\title{
Joint Improvements of Radar/infrared Stealth for Exhaust System of Unmanned Aircraft Based on Sorting Factor Pareto Solution
}

\author{
Zeyang Zhou ( $\sim$ zeyangzhou@buaa.edu.cn ) \\ Beihang University \\ Jun Huang \\ Beihang University
}

\section{Research Article}

Keywords: aircraft exhaust system, electromagnetic scattering, radar cross-section, infrared radiation, Pareto solution, sorting factor

Posted Date: December 31st, 2020

DOl: https://doi.org/10.21203/rs.3.rs-135958/v1

License: (c) (i) This work is licensed under a Creative Commons Attribution 4.0 International License.

Read Full License

Version of Record: A version of this preprint was published at Scientific Reports on April 15th, 2021. See the published version at https://doi.org/10.1038/s41598-021-87756-0. 


\title{
Joint improvements of radar/infrared stealth for exhaust system of unmanned aircraft based on sorting factor Pareto solution
}

\author{
Zeyang ZHOU*, Jun HUANG \\ School of Aeronautic Science and Engineering, Beihang University, Beijing 100083, China
}

\begin{abstract}
In order to reduce the radar cross section (RCS) of the unmanned aircraft while suppressing its infrared signature, a comprehensive design method (CDM) based on sorting factor Pareto solution is presented. The physical optics and physical diffraction theory are used to evaluate the electromagnetic scattering characteristics of the aircraft, and the Monte Carlo and ray tracing method are used to evaluate the infrared radiation intensity of the exhaust system. CDM is used to evaluate and screen each individual in each offspring, and the design parameters and sub-models of the aircraft exhaust system are continuously improved. The results show that the exhaust port model, lower baffle and nozzle height are the main factors affecting the RCS indicators, nozzle stages, exhaust port model, lower baffle and outer width make the main contribution to infrared radiation suppression. The presented CDM is efficient and effective in enhancing the radar/infrared integrated stealth performance of the aircraft.
\end{abstract}

Keywords: aircraft exhaust system; electromagnetic scattering; radar cross-section; infrared radiation; Pareto solution; sorting factor.

\section{Introduction}

The exhaust system of an unmanned fighter not only affects its aerodynamic characteristics and infrared (IR) signature, but also affects the electromagnetic scattering characteristics of its tail and sides [1-3]. Studying the comprehensive design of the exhaust system is of great significance to the infrared/radar stealth performance of the aircraft.

\footnotetext{
${ }^{*}$ Corresponding author.

E-mail address: zeyangzhou @ buaa.edu.cn (Z. ZHOU)
} 
Future fighters and bombers are inclined to ultra-flat fuselage layout, which also brings certain challenges to the design of their intake and exhaust systems, while the balance and restriction between multiple performances is also the key concern of interdisciplinary [4,5]. Computational fluid dynamics (CFD), physical optics (PO), radial basis functions and sequential quadratic programming are integrated to optimize the aerodynamic/stealth characteristics of the flying wing aircraft, and good design variables and strategies are obtained [6]. Diverter less technology and tail nozzle baffles are often used on stealth fighters to improve the aerodynamic and stealth characteristics of these aircraft $[7,8]$. Compared with the traditional layout, the flying wing layout has the advantages of high aerodynamic efficiency, stealth and internal space utilization $[9,10]$, which makes the design of its exhaust system must take into account the low radar cross-section (RCS) and infrared radiation. Surface pixel method, PO and equivalent electromagnetic flow method are used to solve the RCS of three-wing fighter and stealth fighter [11,12]. Based on the existing aerodynamic design concepts and RCS analysis methods, the stealth development of the unmanned fighter exhaust system will pay more attention to infrared radiation suppression and radar/infrared integrated stealth.

The rapid development of high-sensitivity, high-resolution infrared imaging detection technology has made the survivability of fighters with radar stealth design also challenged [13,14]. Replace the axisymmetric nozzle with a binary non-axisymmetric nozzle with larger width and height to obtain better infrared radiation suppression. Combining the exponential broad band model and the radiation transfer equation (RTE), an infrared radiation characteristic estimation method was developed to evaluate the infrared signature of the engine exhaust system [15]. Using the CFD method, the temperature field of the unmanned combat aircraft in the non-forced state is obtained, and the numerical calculation of infrared radiation is performed [16,17]. Ray tracing method is used to solve the infrared signature of high 
temperature exhaust gas from different nozzles [18]. The serpentine nozzle can effectively suppress the infrared radiation characteristics while enhancing the radar stealth characteristics of military aircraft [19]. With the rise of comprehensive stealth research [20], the design and optimization of unmanned combat aircraft are more inclined to consider multiple performance [21,22]. The gray correlation model can effectively evaluate the aerodynamic characteristics, RCS and IR signatures of the aircraft [22-24]. The comprehensive optimization method based on Pareto solution can also give a better solution to the needs of many aspects of stealth $[25,26]$. The continuous development of various technologies of unmanned bombers [27] has also prompted its radar stealth design and infrared radiation suppression to achieve a better balance.

Previous research on the exhaust system of unmanned combat aircraft has mostly focused on aerodynamic shape improvement and infrared radiation characteristic calculation. With the continuous development and joint search of radar and infrared detectors, unilateral reduction of RCS or IR signatures has been unable to counter the threat of multiphysics. In view of the mutual constraints of aerodynamics, radar stealth and infrared radiation suppression when designing the unmanned aircraft exhaust system, this paper attempts to establish a hybrid design approach to establish a comprehensive stealth scheme that takes into account aerodynamic characteristics. This is of guiding significance and engineering value for improving the survivability and integrated combat effectiveness of unmanned fighters.

In this manuscript, the comprehensive design method (CDM) is presented in Section 2. Models of unmanned aircraft and exhaust system are built in Section 3. The results of RCS and IR signatures are provided and discussed in detail in Section 4. Finally, the full article is summarized in Section 5.

\section{Comprehensive design method}

The analysis of the threat of unmanned aircraft from radar and infrared detectors is shown in Fig. 1, 
where $\alpha$ is the azimuth between the radar station and the aircraft, $\beta$ is the elevation angle between the radar station and the aircraft. The comprehensive design method is used to reduce the electromagnetic scattering level of the aircraft exhaust system while suppressing its infrared radiation.

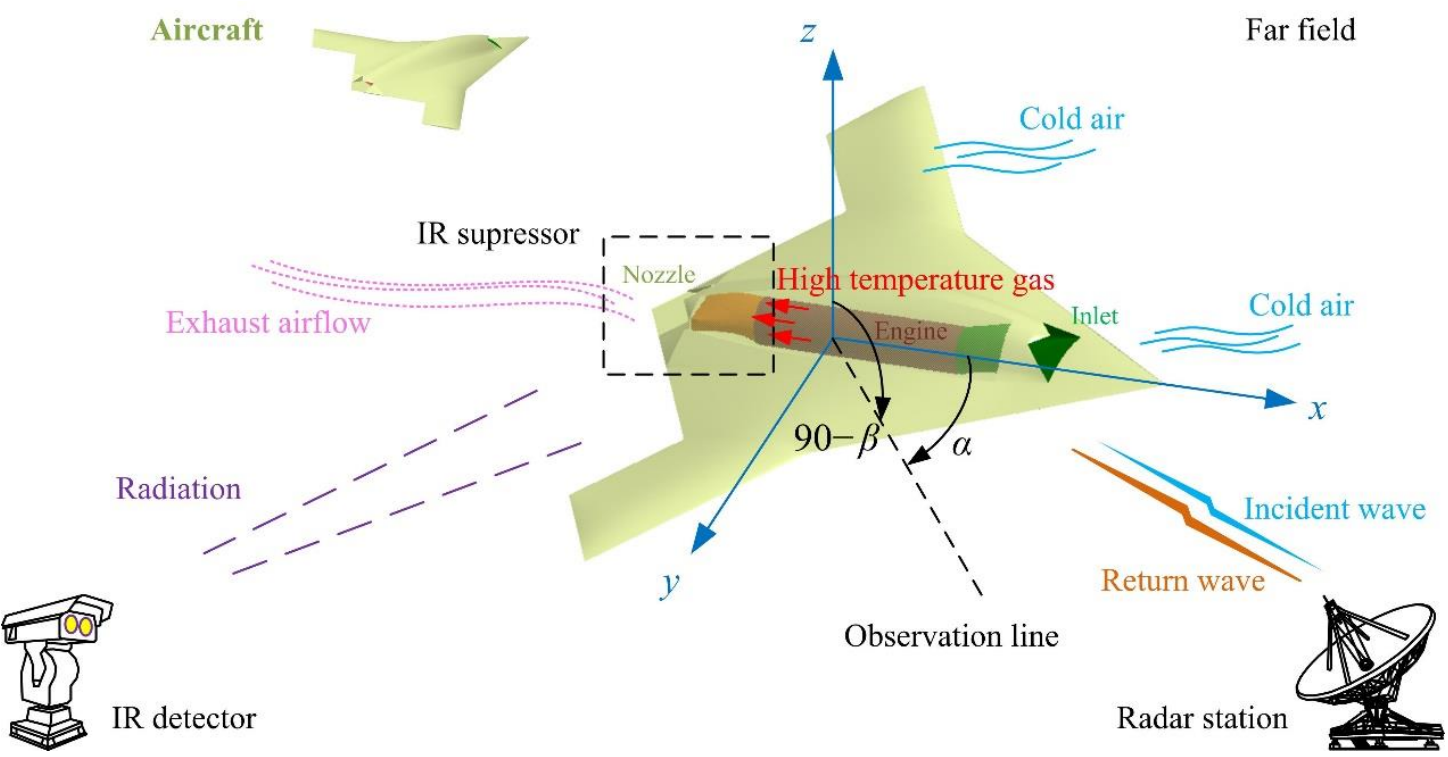

Fig. 1 Schematic diagram of radar/infrared integrated stealth design of aircraft exhaust system.

The entire design process includes three parts as shown in Fig. 2: the establishment of the initial model $\left(m_{0}\right)$, the calculation of performance indicators and the cycle judgment module. The first part emphasizes that both the flow field calculation and the grid need to converge to ensure that the subsequent calculations are comparable. The middle module performs aerodynamic, IR and RCS evaluation on each individual in each offspring, and comprehensively evaluates the optimal solution $\left(M^{*}\right)$. The third part judges the termination of the obtained $M^{*}$ to see if the next generation (Gen) design is needed. 


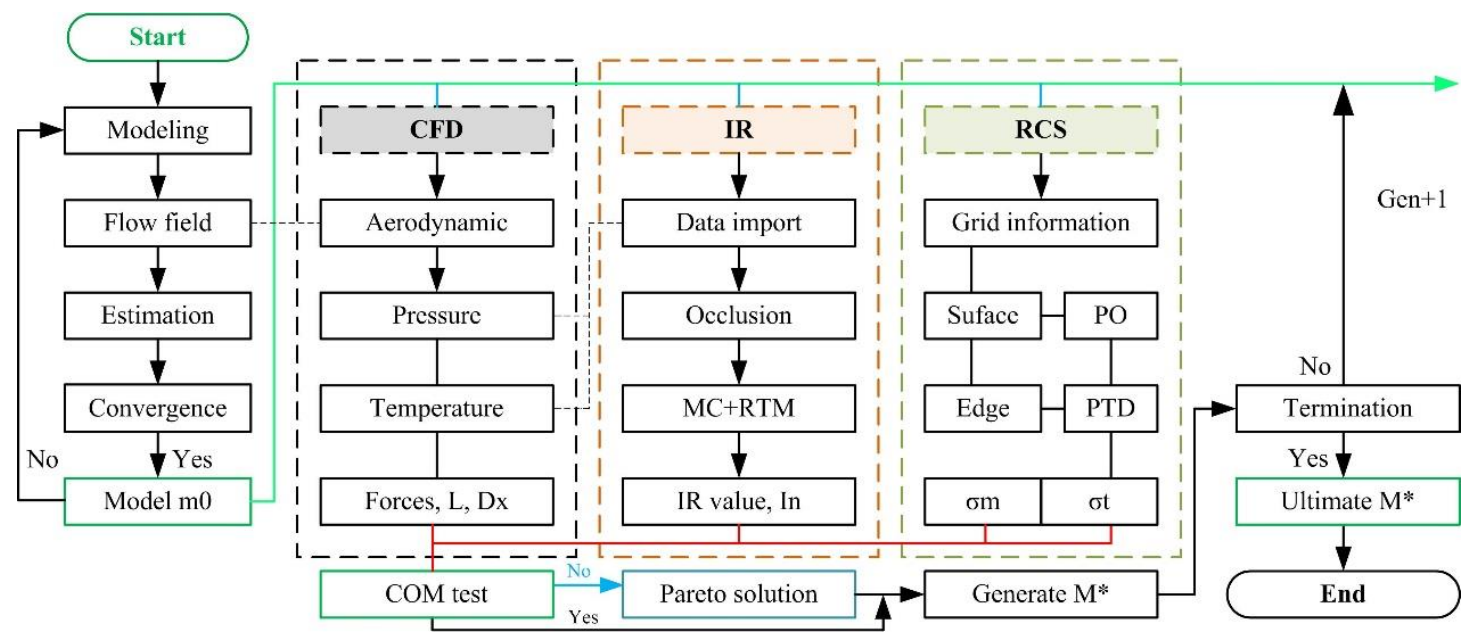

Fig. 2 Flow chart of the comprehensive design method.

\subsection{RCS Calculation}

PO and physical theory of diffraction (PTD) are used to solve the electromagnetic scattering characteristics of each individual in each offspring [3]. According to the magnetic vector position caused by the induced current on the surface of the target model, the electric field and magnetic field can be obtained as follows:

$$
\begin{aligned}
& \boldsymbol{E}(\boldsymbol{r})=\frac{1}{\mathrm{j} \omega \varepsilon \cdot 4 \pi} \iint_{S^{\prime}}\left[\frac{3-k^{2} R^{2}+\mathrm{j} 3 k R}{R^{5}} \mathrm{e}^{-\mathrm{j} k R} \boldsymbol{R} \times\left(\boldsymbol{R} \times \boldsymbol{J}_{\mathrm{s}}\left(\boldsymbol{r}^{\prime}\right)\right)+2 \boldsymbol{J}_{\mathrm{s}}\left(\boldsymbol{r}^{\prime}\right) \frac{1+\mathrm{j} k R}{R^{3}} \mathrm{e}^{-\mathrm{j} k R}\right] \mathrm{d} S^{\prime} \\
& \boldsymbol{H}(\boldsymbol{r})=\frac{1}{4 \pi} \iint_{S^{\prime}} \frac{-1-\mathrm{j} k R}{R^{3}} e^{-\mathrm{j} k R}\left(\boldsymbol{R} \times \boldsymbol{J}_{\mathrm{s}}\left(\boldsymbol{r}^{\prime}\right)\right) \mathrm{d} S^{\prime}
\end{aligned}
$$

where $\boldsymbol{r}$ is the coordinate vector of the field point, $\omega$ refers to the electromagnetic wave angular frequency, $k$ represents the wave number in free space, $\boldsymbol{R}$ is the distance vector between the field point and the source point, $\boldsymbol{r}^{\prime}$ refers to the coordinate vector of the source point, $\varepsilon$ is the dielectric permittivity [28-30], $\boldsymbol{J}_{\mathrm{s}}$ represents the induced current on the target surface.

According to the assumption of PO, there is current in the illuminated area of the target surface, but no current in the dark area:

$$
\boldsymbol{J}_{\mathrm{s}}= \begin{cases}2 \boldsymbol{n} \times \boldsymbol{H} & Z_{\mathrm{I}} \\ \mathbf{0} & Z_{\mathrm{D}}\end{cases}
$$

where $Z_{\mathrm{I}}$ refers to the illuminated area, $Z_{\mathrm{D}}$ is the dark area, and $\boldsymbol{n}$ represents the unit normal vector of the 
outer normal direction of $\boldsymbol{r}^{\prime}$ at the surface of the scatterer. Therefore, the electric field formula can be changed to

$$
\boldsymbol{E}^{s}(\boldsymbol{r})=\frac{\mathrm{j}}{\lambda \boldsymbol{r}}\left|\boldsymbol{E}_{0}\right| \mathrm{e}^{-\mathrm{j} k \cdot \boldsymbol{r}} \iint_{S^{\prime}} \hat{\boldsymbol{r}} \times\left\{\hat{\boldsymbol{r}} \times\left[\left(\hat{\boldsymbol{n}}\left(\boldsymbol{r}^{\prime}\right) \cdot \boldsymbol{E}_{0}\right) \hat{\boldsymbol{k}}-\left(\hat{\boldsymbol{n}}\left(\boldsymbol{r}^{\prime}\right) \cdot \hat{\boldsymbol{k}}\right) \boldsymbol{E}_{0}\right]\right\} \mathrm{e}^{-\mathrm{j} k(-\hat{r}+\hat{k}) \cdot \boldsymbol{r}^{\prime}} \mathrm{d} S^{\prime}
$$

where $\boldsymbol{k}$ means wave vector, $\lambda$ represents the wavelength in free space, $r$ refers to the distance from the field point to the origin of coordinates. The integral term can be calculated separately, so there is the following expression:

$$
I=\iint_{S^{\prime}} \hat{\boldsymbol{r}} \times\left\{\hat{\boldsymbol{r}} \times\left[\left(\hat{\boldsymbol{n}}\left(\boldsymbol{r}^{\prime}\right) \cdot \boldsymbol{E}_{0}\right) \hat{\boldsymbol{k}}-\left(\hat{\boldsymbol{n}}\left(\boldsymbol{r}^{\prime}\right) \cdot \hat{\boldsymbol{k}}\right) \boldsymbol{E}_{0}\right]\right\} \mathrm{e}^{-\mathrm{j} k(-\hat{\boldsymbol{r}}+\hat{\hat{k}}) \cdot \boldsymbol{r}^{\prime}} \mathrm{d} S^{\prime}
$$

Therefore, the RCS of the surface can be determined as

$$
\sigma_{\mathrm{PO}}=\frac{4 \pi}{\lambda^{2}}|I|^{2}
$$

In fact, the aircraft model has many edges and split angles, and these geometric features will also produce diffraction effects on electromagnetic waves. Here, PTD is used to solve the edge diffraction. ${ }^{20}$ The total RCS can be expressed as the joint contribution of PO and PTD:

$$
\sigma=\left|\sum_{i=1}^{N_{\mathrm{F}}}\left(\sqrt{\sigma_{\mathrm{PO}}}\right)_{i}+\sum_{j=1}^{N_{\mathrm{E}}}\left(\sqrt{\sigma_{\mathrm{PTD}}}\right)_{j}\right|^{2}
$$

where $N_{\mathrm{F}}$ refers to the number of facets and $N_{\mathrm{E}}$ represents the number of edges. The presented RCS calculation method is verified by PO+MOM (Method of Moment)/MLFMM (Multi-Level Fast Multipole Method) in FEKO (FEldberechnung bei Korpern mit beliebiger Oberflache) as shown in Fig. 3, where $f_{\mathrm{R}}$ is the radar wave frequency and the radar wave uses horizontal polarization. It can be seen that the two RCS curves are roughly coincident, and the result calculated by FEKO is slightly larger than the other, where the mean of the RCS curve of PO+PTD is smaller than that of FEKO by $0.3909 \mathrm{dBm}^{2}$. This is because the two use different grid processing methods and different RCS algorithms. These results indicate that the RCS algorithm presented in this paper is accurate to deal with the electromagnetic scattering characteristics of the aircraft model. 


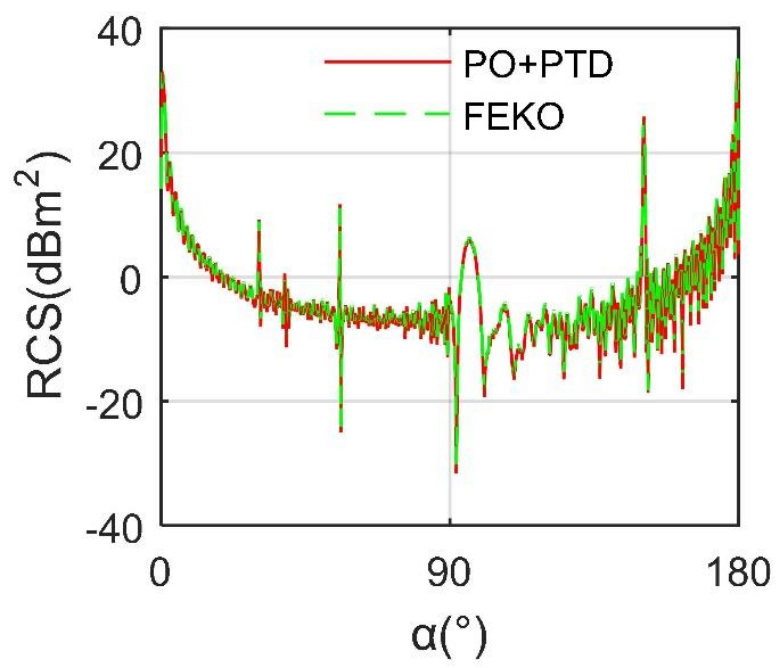

Fig. 3 Verification of RCS calculation method on $m_{0}, \beta=0^{\circ}, f_{\mathrm{R}}=10 \mathrm{GHz}$.

\subsection{IR signature evaluation}

Monte Carlo (MC) and ray tracing method (RTM) are used to solve the infrared signature of aircraft exhaust system [3]. Consider the absorption and emission in the RTE, without considering the scattering of the medium, so that the refractive index is always equal to 1, then RTE can be described as:

$$
\frac{\mathrm{d} L(\boldsymbol{r}, \boldsymbol{s})}{\mathrm{d} s}+a L(\boldsymbol{r}, \boldsymbol{s})=a \frac{\sigma T^{4}}{\pi}
$$

where $\boldsymbol{r}$ refers to the position vector, $\boldsymbol{s}$ is the direction vector, $s$ represents the length along the path, $a$ is the absorption coefficient, $L$ is the radiance, $\sigma$ refers to the Stefan-Boltzmann constant, $T$ is the local temperature.

Using discrete coordinate model to calculate thermal radiation, RTE can be transformed into:

$$
\nabla \cdot[L(\boldsymbol{r}, \boldsymbol{s}) \boldsymbol{s}]+a L(\boldsymbol{r}, \boldsymbol{s})=a \frac{\sigma T^{4}}{\pi}
$$

According to MC+RTM, the spectral radiance reaching the receiving point of the detector can be expressed as:

$$
L_{\sigma}=L_{\sigma}^{0} \tau_{1 \sigma} \tau_{2 \sigma} \cdots \tau_{n \sigma}+L_{b \sigma}^{1}\left(1-\tau_{1 \sigma}\right) \tau_{2 \sigma} \tau_{3 \sigma} \cdots \tau_{n \sigma}+\cdots+L_{b \sigma}^{n-1}\left(1-\tau_{(n-1) \sigma}\right) \tau_{n \sigma}+L_{b \sigma}^{n}\left(1-\tau_{n \sigma}\right)
$$

where $L_{\sigma}^{0}$ refers to spectral radiance of wall reverse rays, $L_{b \sigma}^{i}$ means spectral radiance, $\tau_{i \sigma}$ represents $i$-th layer spectral transmittance. 
On this basis, the irradiance reaching the receiving point of the detector can be calculated as:

$$
E=\sum_{i=1}^{N_{\mathrm{b}}} \sum_{j=1}^{N} L_{\sigma,(i, j)}^{n} \cdot \cos \theta_{j} \cdot \Delta \Omega_{j} \cdot \Delta \sigma_{i} \cdot 100
$$

where $E$ means the radiant illumination, $N_{\mathrm{b}}$ refers to the total number of wave bands, $N$ represents the total number of rays contributing to the measurement point, $\theta_{j}$ refers to the angle between the center of the $j$-th solid angle and the surface normal of its measuring point, $\Delta \Omega_{j}$ means the $j$-th solid angle, $\Delta \sigma_{i}$ is the width of the $i$-th wave band.

At this time, the radiation intensity can be determined as:

$$
I_{\sigma}=E_{\sigma}(R) \cdot R^{2}
$$

where $I$ is the radiation intensity and $R$ refers to the linear distance between the aircraft and the IR detector.

For the infrared radiation of the exhaust pipe, regarding the exhaust pipe as a gray body, the total radiation and total radiation intensity can be expressed as:

$$
\begin{aligned}
& L_{\Delta \lambda}=\frac{\varepsilon}{\pi} M_{\lambda_{1} \sim \lambda_{2}}=\frac{\varepsilon}{\pi} \int_{\lambda_{1}}^{\lambda_{2}} M_{\lambda} \mathrm{d} \lambda \\
& I_{\Delta \lambda}=L_{\Delta \lambda} \Delta A \cos \theta
\end{aligned}
$$

where $\lambda$ is the wavelength, $M_{\lambda_{1} \sim \lambda_{2}}$ represents the radiation emission degree of the black body between the bands $\lambda_{1} \sim \lambda_{2}, \Delta A$ is the area of the nozzle bin, $M_{\lambda}$ means the spectral radiation emission degree of the black body.

Noting the Planck's formula:

$$
M_{\lambda}=\frac{c_{1}}{\lambda^{5}} \frac{1}{e^{c_{2} /(\lambda T)}-1}
$$

Thus the radiation emittance of blackbody in $\lambda_{1} \sim \lambda_{2}$ band could be calculated as:

$$
M_{\lambda_{1} \sim \lambda_{2}}=\frac{15 \sigma T^{4}}{\pi^{4}} \int_{c_{2} /\left(\lambda_{2} T\right)}^{c_{2} /\left(\lambda_{1} T\right)} \frac{x^{3}}{e^{x}-1} \mathrm{~d} x
$$

The radiation constants have the following relationship:

$$
c_{1}=2 \pi h c^{2}, \quad c_{2}=\frac{h c}{k}, \quad \sigma=\frac{2 \pi^{5} k^{4}}{15 c^{2} h^{3}}
$$


where $h$ refers to the Planck constant, $h=6.626176 \times 10^{-34} \mathrm{~J} \cdot \mathrm{s}, k=1.38 \times 10^{-23} \mathrm{~J} / \mathrm{K}, c$ is the speed of light. The IR signature calculation method presented here is verified as shown in Fig. 4, where the calculation results presented here are basically consistent with the known data in the literature [26]. The mean of the red data is $1.67 \mathrm{~W} / \mathrm{sr}$ smaller than that of the green data. These results indicate that the IR calculations in this paper are feasible and accurate for evaluating the infrared radiation characteristics of this aircraft exhaust system.

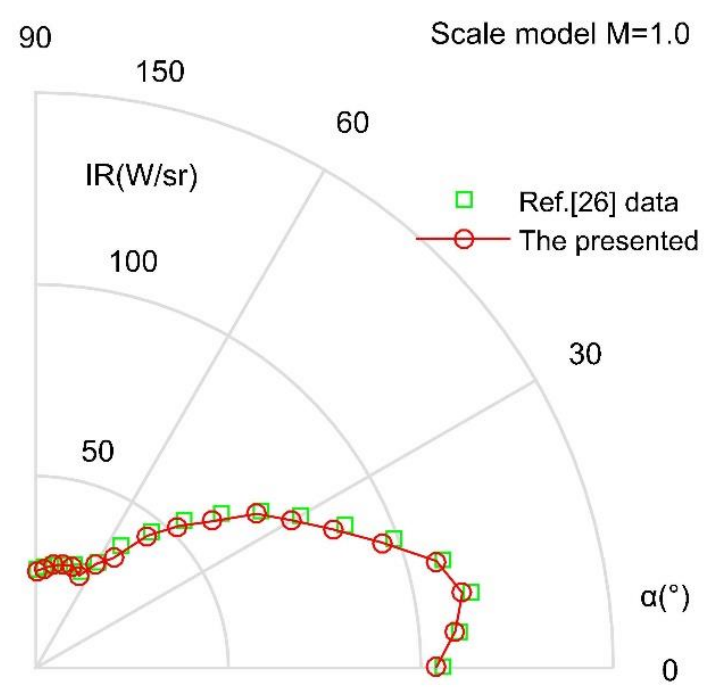

Fig. 4 Verification of IR signature calculation method, 3-5 $\mu$ m wavelength band.

\subsection{Comprehensive stealth design}

In order to improve the radar/infrared stealth performance of this aircraft, the objective function is defined as follows:

$$
\left\{\begin{array}{l}
\min : f_{i}(m), \quad i=1,2,3 \\
M=\left\{m_{1}, m_{2}, \ldots, m_{n}\right\}
\end{array}\right.
$$

where $i$ is the serial number, $m$ refers to the individual of the aircraft model, $M$ is the collection of descendants $[20,25]$. The comprehensive stealth indicators are:

$$
\left\{\begin{array}{l|l}
f_{1}(m)=\sigma_{\mathrm{m}} & 0^{\circ} \leq \alpha \leq 180^{\circ}, \quad \beta=0^{\circ} \\
f_{2}(m)=\sigma_{\mathrm{t}} & f_{\mathrm{R}}=10 \mathrm{GHz}, \quad \mathrm{HH} \\
f_{3}(m)=I_{\mathrm{n}} & -30^{\circ} \leq \alpha_{n} \leq 30^{\circ}
\end{array}\right.
$$


where $\mathrm{HH}$ represents that the radar wave is horizontally polarized, $\alpha_{n}$ means the observation angle in the normal observation field, $\sigma_{\mathrm{m}}$ is the mean RCS indicator, $\sigma_{\mathrm{t}}$ is the tail RCS indicator, $I_{\mathrm{n}}$ is the infrared radiation intensity indicator in the normal observation field.

Throughout the overall design process, equation constraints are expressed as follows:

$$
\left\{\begin{array} { l } 
{ W _ { \mathrm { f } } ( M _ { i } ) - W _ { \mathrm { f } } ( M _ { j } ) = 0 } \\
{ L _ { \mathrm { e } } ( M _ { i } ) - L _ { \mathrm { e } } ( M _ { j } ) = 0 } \\
{ X _ { \mathrm { n } } ( M _ { i } ) - X _ { \mathrm { n } } ( M _ { j } ) = 0 }
\end{array} \quad \& \quad \left\{\begin{array}{l}
D_{\text {in }}\left(M_{i}\right)-D_{\text {in }}\left(M_{j}\right)=0 \\
L_{\text {in }}\left(M_{i}\right)-L_{\text {in }}\left(M_{j}\right)=0 \\
X_{\text {in }}\left(M_{i}\right)-X_{\text {in }}\left(M_{j}\right)=0
\end{array} \mid \forall i, j \in\{1,2, \ldots, k\}\right.\right.
$$

where $W_{\mathrm{f}}$ refers to the width of the aircraft fuselage, $D_{\mathrm{in}}$ is the diameter of the end of the intake pipe, $L_{\mathrm{e}}$ is the length of the engine compartment, $L_{\text {in }}$ represents the length of the air intake, $X_{\mathrm{n}}$ means the $x$ coordinate of the front face of the nozzle, $X_{\text {in }}$ refers to the $x$ coordinate of the end face of the intake pipe, and $k$ is the number of generations.

The inequality equality constraints can be expressed as:

$$
\left\{\begin{array} { l } 
{ L _ { \mathrm { f } } ( M _ { i } ) - L _ { \mathrm { f } , \operatorname { m a x } } \leq 0 } \\
{ L _ { \mathrm { ex } } ( M _ { i } ) - L _ { \mathrm { ex } , \operatorname { m a x } } \leq 0 }
\end{array} \& \left\{\begin{array}{l|l}
D_{x}\left(M_{j+1}\right)-D_{x}\left(M_{j}\right) \leq 0 & \forall i \in\{1,2, \ldots, k\} \\
T_{\mathrm{n}}\left(M_{j+1}\right)-T_{\mathrm{n}}\left(M_{j}\right) \leq 0 & \forall j \in\{1,2, \ldots, k-1\}
\end{array}\right.\right.
$$

where $L_{\mathrm{f}}$ refers to the length of the fuslage, $L_{\mathrm{ex}}$ is the length of the exhaust pipe, $L_{\mathrm{f}, \max }$, and $L_{\mathrm{ex}, \max }$ are the limit values, $D_{\mathrm{x}}$ is the drag indicator, $T_{\mathrm{n}}$ is the temperature indicator of nozzle face.

When there are optimal solutions for the stealth indicators, the following relationship (COM test [20,25]) should be satisfied:

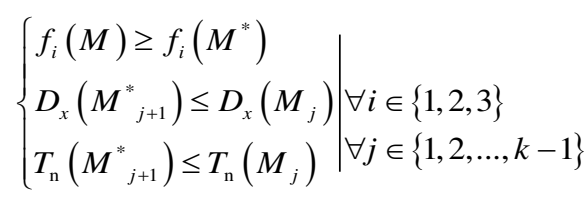

When the optimal solution does not exist, that is, these stealth indicators cannot reach the minimum at the same time, then the Pareto solution needs to be applied:

$$
\left\{\begin{array}{l}
f_{i}\left(M^{*}\right) \leq f_{i}(M) \\
D_{x}\left(M_{j}\right) \geq D_{x}\left(M^{*}{ }_{j+1}\right) \\
T_{\mathrm{n}}\left(M_{j}\right) \geq T_{\mathrm{n}}\left(M^{*}{ }_{j+1}\right)
\end{array} \mid \begin{array}{l}
\exists i \in\{1,2,3\} \\
\exists j \in\{1,2, \ldots, k-1\}
\end{array}\right.
$$


In order to establish individuals with better stealth performance, a ranking factor can be defined here to distinguish these feasible solutions in each generation:

$$
\begin{aligned}
& f_{\mathrm{s}}(i, j)=r_{\text {ind }}(i, j) / N_{\text {ind }} \\
& f_{r}(i)=\sum_{j=1}^{N_{\text {indc }}} f_{\mathrm{s}}(i, j) / N_{\text {indc }}
\end{aligned}
$$

where $f_{\mathrm{s}}(i, j)$ represents the sorting factor of the $j$-th stealth indicator of the $i$-th individual, $N_{\text {ind }}$ is the number of individuals in the current offspring, $r_{\text {ind }}(i, j)$ indicates the ascending ranking of the $j$-th indicator of the $i$-th individual, $f_{\mathrm{r}}$ is the ranking factor, $N_{\text {indc }}$ is the number of stealth indicators.

At this time, the optimal solution can be expressed as the individual with the smallest $f_{\mathrm{r}}$ :

$$
M^{*}=m_{i} \mid \min \left(f_{\mathrm{r}}(i)\right)
$$

If the above ranking factor Pareto section is still not unique, the priority is defined to continue to distinguish these individuals:

$$
p_{\text {indc }}\left(\sigma_{\mathrm{m}}\right) \geq p_{\text {indc }}\left(\sigma_{\mathrm{t}}\right) \geq p_{\text {indc }}\left(I_{\mathrm{n}}\right)
$$

where $p_{\text {inde }}$ is the priority of individual stealth indicator. At this time, priority is given to the individual whose RCS mean indicator performs better.

\section{Model}

The outline design of the aircraft symmetry plane is shown in Fig. 5, where $M_{\text {ex }}$ represents the model of the exhaust port, $B_{\mathrm{d}}$ is the nozzle model with downward blocking, $N_{\mathrm{s}}$ refers to the number of nozzle stages, $B_{\mathrm{u}}$ is a nozzle model with upward blocking, $H_{\mathrm{n}}$ is the height of the nozzle. 


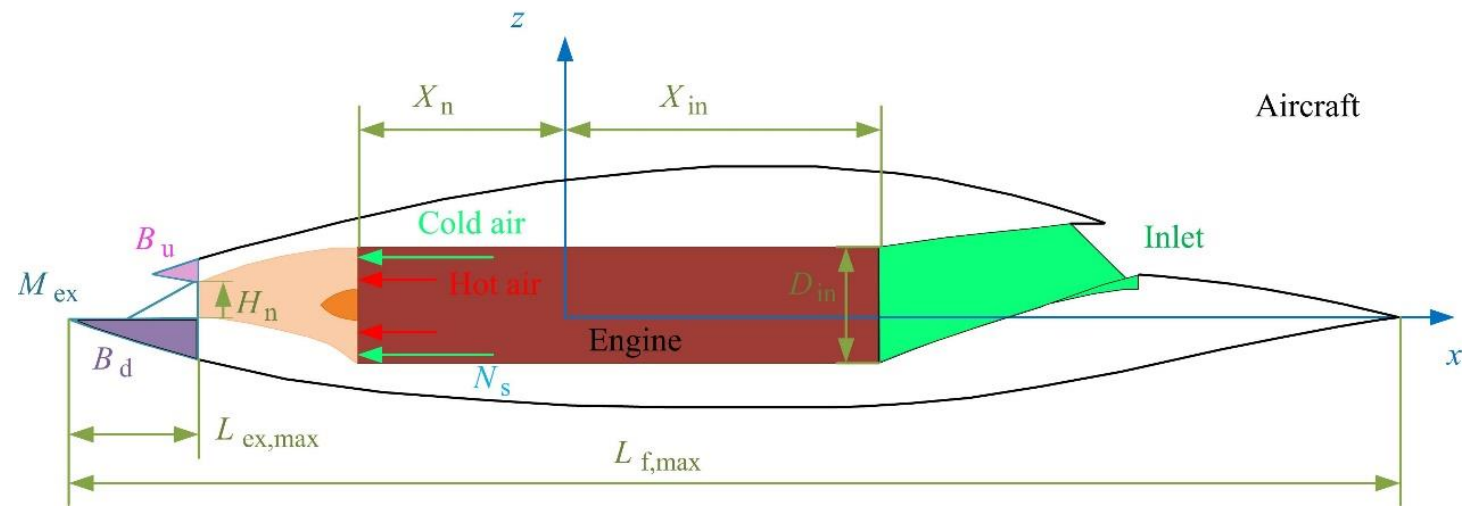

Fig. 5 Aircraft design draft and parameter variables.

The model of the unmanned aircraft was built as shown in Fig. 6, where $W_{\mathrm{n}}$ is a custom parameter used to determine the nozzle width, $X_{\mathrm{v}}$ is a custom length parameter used to determine the size of $B_{\mathrm{d}}$, noting that the outer edge of $B_{\mathrm{d}}$ is parallel to the tail edge of the aircraft. $X_{\mathrm{u}}$ is the size parameter of $B_{\mathrm{u}}$. When the parameters of the exhaust system change, the aircraft model also changes, and the aircraft model here can be represented by the following parameters or sub-models:

$$
m=\left\{M_{\mathrm{ex}}, N_{\mathrm{s}}, B_{\mathrm{d}}, H_{\mathrm{n}}, W_{\mathrm{n}}, B_{\mathrm{u}}\right\}
$$



Fig. 6 Three-view display of aircraft geometric model.

The flow field of the aircraft exhaust system is established as shown in Fig. 7, where high-precision unstructured grid technology is used to divide the external, central and internal flow field areas. Mesh 
encryption is added to surfaces and edges with small dimensions or large curvature changes, including air intakes, exhaust ports, center body, nozzle pipe, leading and trailing edges of wings. The velocity of the incoming flow in the far front of the aircraft is set to $85 \mathrm{~m} / \mathrm{s}$ with the temperature $300 \mathrm{~K}$, while the high-temperature airflow at the nozzle inlet is set at $870 \mathrm{~K}$. The standard $k$ - $\varepsilon$ model is used to solve the Navier-Stocks equation of the exhaust system flow field. Momentum, density, turbulent kinetic energy, energy and turbulent dissipation rate are discretized using a second-order upwind scheme.
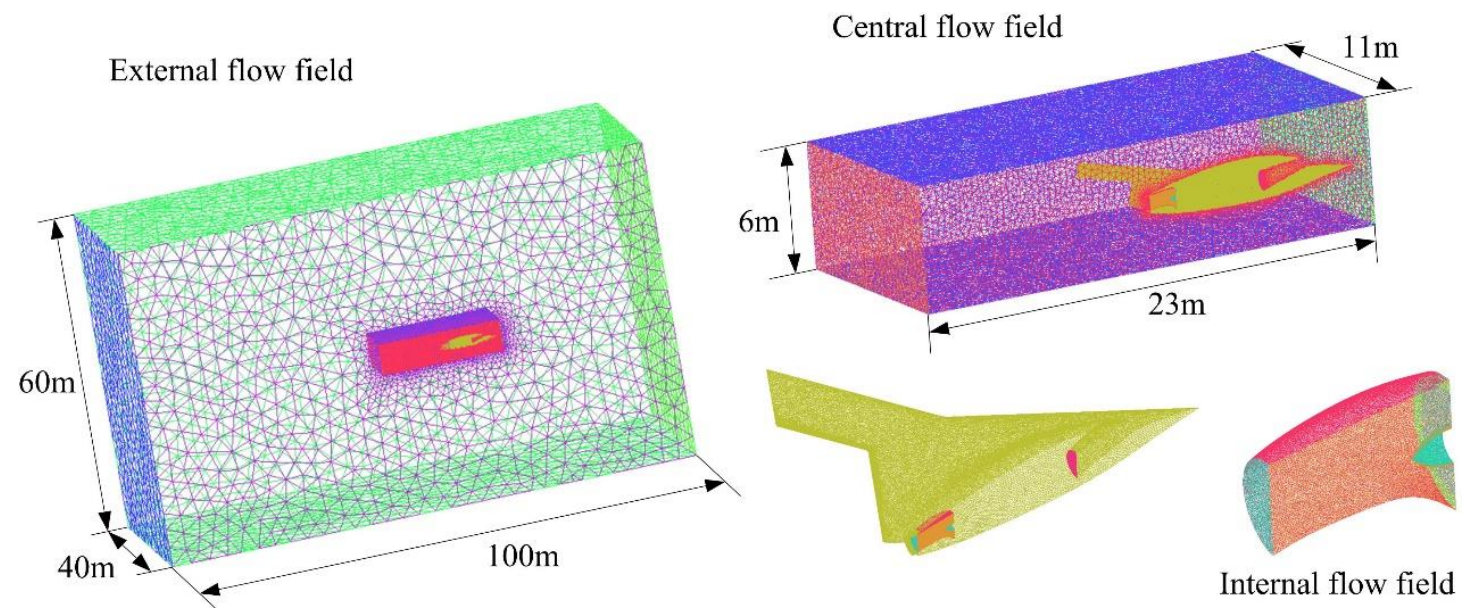

Fig. 7 Aircraft exhaust system flow field construction and grid division.

In order to evaluate the changes in stealth characteristics brought to the aircraft during the design of the exhaust system, the radar and infrared observation fields are set as shown in Fig. 8, where $\sigma_{\mathrm{m}}$ is equal to the mean RCS in the range of $0^{\circ} \leq \alpha \leq 180^{\circ}, \sigma_{\mathrm{t}}$ is equal to mean RCS in the range of $150^{\circ} \leq \alpha \leq 180^{\circ}, I_{\mathrm{n}}$ is equal to the mean value of the IR radiation intensity in the range of $-30^{\circ} \leq \alpha_{\mathrm{n}} \leq 30^{\circ}$.


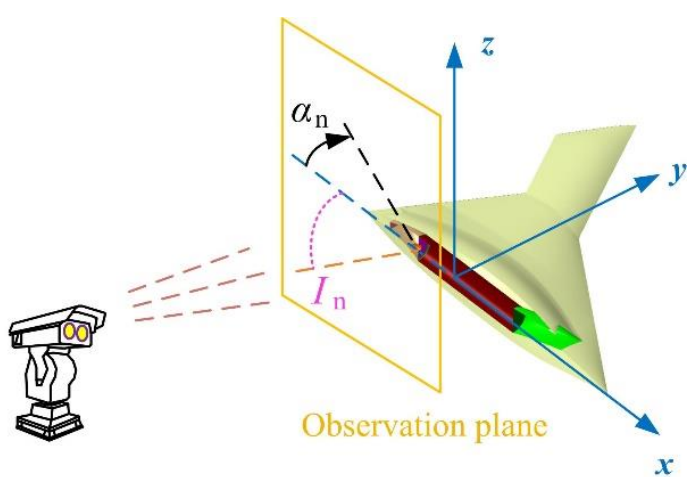

IR detector 
Fig. 8 Observation field setting of aircraft radar cross section and IR signature, $\beta=0^{\circ}$.

\section{Results and discussion}

Fig. 9 presents that the aircraft's RCS and RCS mean indicators show great differences under different radar wave frequencies. When $f_{\mathrm{R}}$ is increased from $6 \mathrm{GHz}$ to $10 \mathrm{GHz}$, the RCS curve is generally consistent, but the local amplitude slightly increases, which is very obvious in the peaks of the head, side and tail. The increasing speed of the RCS mean index gradually decreases with the increase of the radar wave frequency. The $\sigma_{\mathrm{m}}$ at $2 \mathrm{GHz}$ is only around $7 \mathrm{dBm}^{2}$, and the index at $12 \mathrm{GHz}$ reaches $13.8551 \mathrm{dBm}^{2}$. In order to make the calculation results of the electromagnetic scattering level of the aircraft comparable in the design process, the radar wave frequency here is set to $10 \mathrm{GHz}$.



(a) RCS

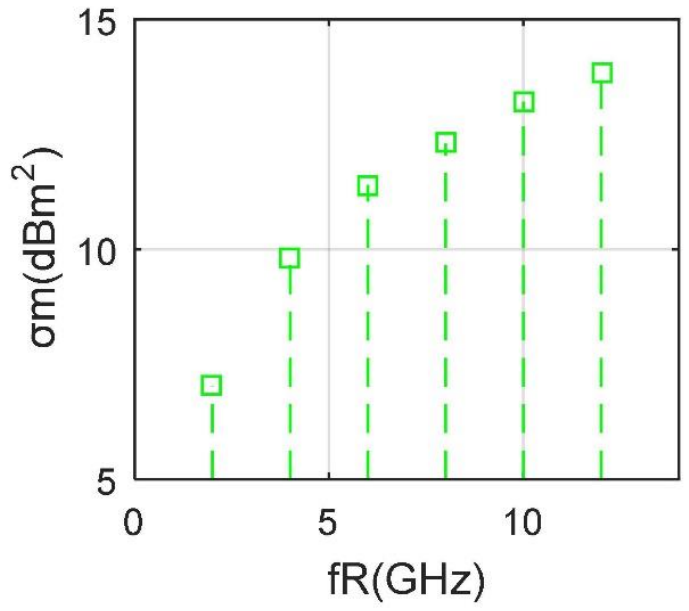

(b) mean RCS

Fig. 9 RCS of aircraft model under different radar wave frequencies, $\beta=0^{\circ}$.

\subsection{Effects of $M_{e x}$ and $N_{s}$}

Fig. 10 provides that these three exhaust port models have a great influence on the RCS of the aircraft, where $M_{\mathrm{ex} 1}$ uses a simple round pipe, $M_{\mathrm{ex} 2}$ uses an oval design, and $M_{\mathrm{ex} 3}$ uses a rectangular nozzle with a triangular upper and lower baffle. It can be seen that the RCS level of $M_{\mathrm{ex} 3}$ is lower than the other two, but it produces a peak of $24.41 \mathrm{dBm}^{2}$ at $144.5^{\circ}$ because $M_{\mathrm{ex} 3}$ does not use the arc design of the first two, but accepts a more concise triangle + large acute angle nozzle, this measure can effectively deflect 
most radar waves to non-threatening azimuth. The RCS curves of $M_{\mathrm{ex} 1}$ and $M_{\mathrm{ex} 2}$ are generally similar, but the peaks and fluctuations in the lateral $92.25^{\circ} \sim 116.5^{\circ}$ are different, where the average RCS of $M_{\mathrm{ex} 2}$ curve is $13.1265 \mathrm{dBm}^{2}$, and that of $M_{\mathrm{ex} 3}$ is $11.6694 \mathrm{dBm}^{2}$. These results show that the reasonable design of the nozzle can effectively reduce the average RCS of the aircraft and the peak RCS of certain azimuths.

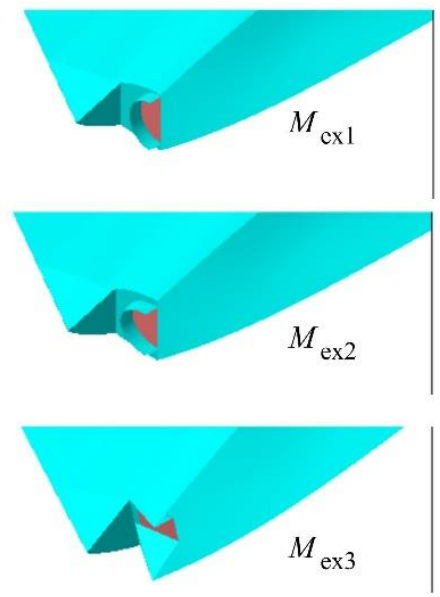

(a) $M_{\mathrm{ex}}$
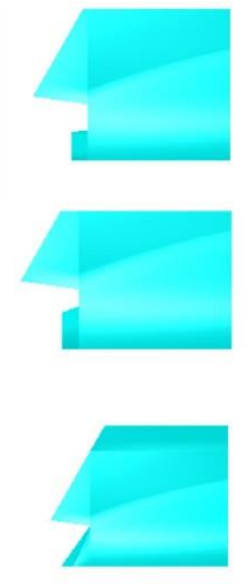

Fig. 11 shows that the IR signatures of aircraft in different bands are very different, and the radiation intensity in the $3 \sim 5 \mu \mathrm{m}$ band is significantly higher than the other two bands. In order to make the infrared radiation index results in the entire comprehensive design process comparable, the following IR calculations are performed in the $3 \sim 5 \mu \mathrm{m}$ band. For the $M_{\mathrm{ex} 3}$, the IR value in the positive observation angle range is significantly larger than that in the negative observation angle range, because the size of the upper baffle of the nozzle is smaller than that of the lower baffle, and the effect of blocking the heat source is small. The maximum IR value when $N_{\mathrm{s}}=1$ reaches $756.41 \mathrm{~W} / \mathrm{sr}$, while the IR curve at $N_{\mathrm{s}}=2$ as a whole is much smaller than the IR curve at $N_{\mathrm{s}}=1$, because at this time the introduction of circular cold air can better surround the high-temperature heat flow and form a certain isolation and protection, these combined effects greatly reduce the IR performance. These results show that the nozzle based on the 
principle of two-stage ejection can significantly improve the infrared radiation characteristics of the aircraft.

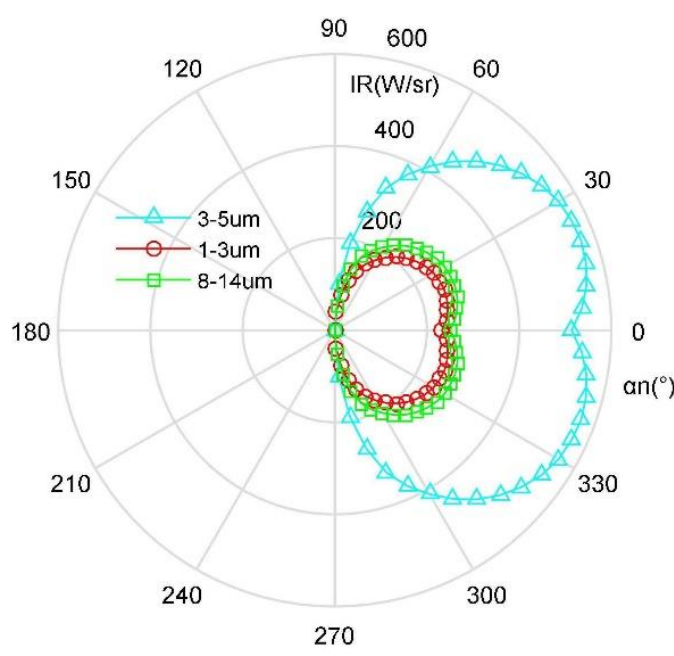

(a) $m_{0}, N_{\mathrm{s}}=1$

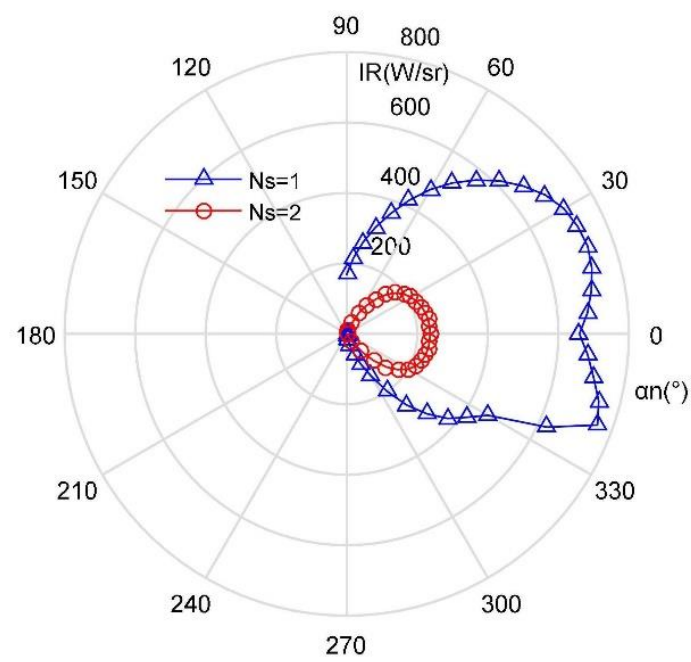

(b) $M_{\mathrm{ex} 3}, 3 \sim 5 \mu \mathrm{m}$

Fig. 11 Aircraft IR signature under different wave bands and $N_{\mathrm{s}}$.

4.2 Effects of $B_{d}$ and $H_{n}$

Fig. 12 provides that the effect of different $M_{\mathrm{bd}}$ models on the stealth performance of the aircraft is obvious. As the value of $X_{\mathrm{v}}$ increases, the size of the lower baffle of the nozzle becomes larger and gradually integrates with the aircraft. This design results in a more concise shape of the entire aircraft and a stable deflection effect on electromagnetic waves, thus the RCS curves of $M_{\mathrm{bd} 2}, M_{\mathrm{bd} 3}$, and $M_{\mathrm{bd} 4}$ are very similar. The RCS curve of $M_{\mathrm{bd} 1}$ has a peak of $24.41 \mathrm{dBm}^{2}$ at $144.5^{\circ}$, and the last three RCS curves have peaks at $150.5^{\circ}$. For the IR curves, the IR curve clearly shows an upward contraction trend with the increase of $X_{v}$, this is because the outward extension of the lower baffle effectively blocks the radiation characteristics in the negative observation angle. As shown in Table 1, the difference between the radar stealth indicators under these four $M_{\mathrm{bd}}$ models is relatively small, but the IR indicator has a significant decreasing trend with a reduction of $65.5709 \mathrm{~W} / \mathrm{sr}$. These results indicate that the design of $M_{\mathrm{bd}}$ contributes more to the infrared stealth of the aircraft. 




(a) RCS

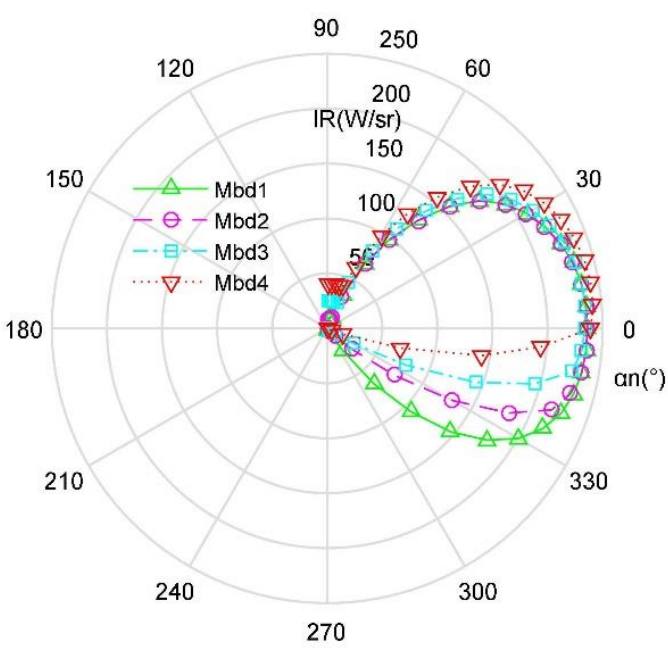

(b) IR curve

Fig. 12 Comparison of stealth characteristics of aircraft under different $M_{\mathrm{bd}}$ models.

Table 1 Comparison of stealth indicators of aircraft under different $M_{\mathrm{bd}}$ models

\begin{tabular}{ccccc}
\hline$M_{\mathrm{bd}}$ & 1 & 2 & 3 & 4 \\
\hline$X_{\mathrm{v}}(\mathrm{mm})$ & 500 & 650 & 850 & 1150 \\
$\sigma_{\mathrm{m}}\left(\mathrm{dBm}^{2}\right)$ & 11.6694 & 11.0772 & 11.1147 & 11.0857 \\
$\sigma_{\mathrm{t}}\left(\mathrm{dBm}^{2}\right)$ & 12.3457 & 12.6437 & 12.5575 & 12.5467 \\
$I_{\mathrm{n}}(\mathrm{W} / \mathrm{sr})$ & 223.7332 & 212.3998 & 187.0830 & 158.1623 \\
\hline
\end{tabular}

Fig. 13 indicates that the height of the exhaust port will also have a more obvious impact on the stealth performance of the aircraft, where $H_{\mathrm{n} 1}=330 \mathrm{~mm}, H_{\mathrm{n} 2}=300 \mathrm{~mm}$. For the IR curves, the IR value of $H_{\mathrm{n} 1}$ is obviously greater than the value of $H_{\mathrm{n} 2}$ except the observation angle is greater than $45^{\circ}$, because reducing the height of the nozzle can increase the mixing intensity of the hot and cold air flow, which results in the normal infrared radiation effect of the nozzle being suppressed, but the blocking effect of the upper baffle on the nozzle is slightly weakened, thus the infrared radiation of the nozzle didn't weaken much when the observation angle increases positively. Note that the IR indicator at $H_{\mathrm{n} 1}$ is around 158 W/sr, while that at $H_{\mathrm{n} 2}$ is reduced to $140.0023 \mathrm{~W} / \mathrm{sr}$. The RCS curves under the two $H_{\mathrm{n}}$ are very similar, where the mean indicator here differs by only $0.1285 \mathrm{dBm}^{2}$. These results indicate that choosing a suitable nozzle height is beneficial to improve the radar/infrared performance of the aircraft. 


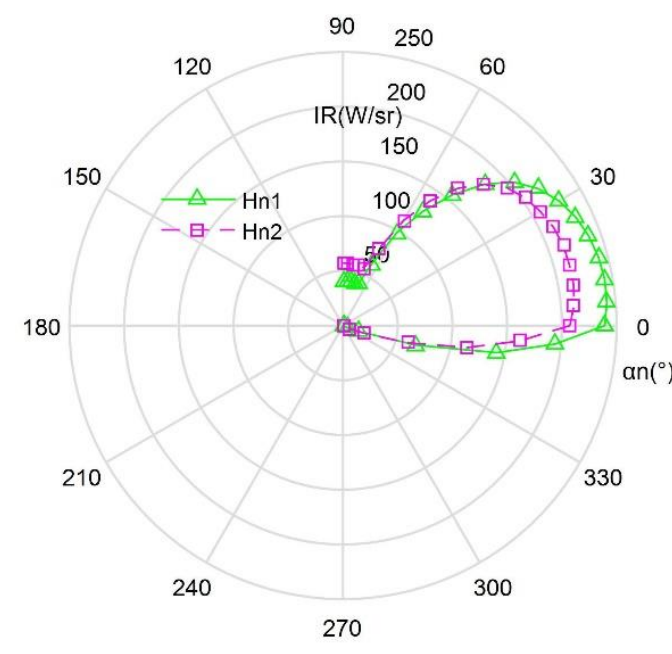

(a) IR curve

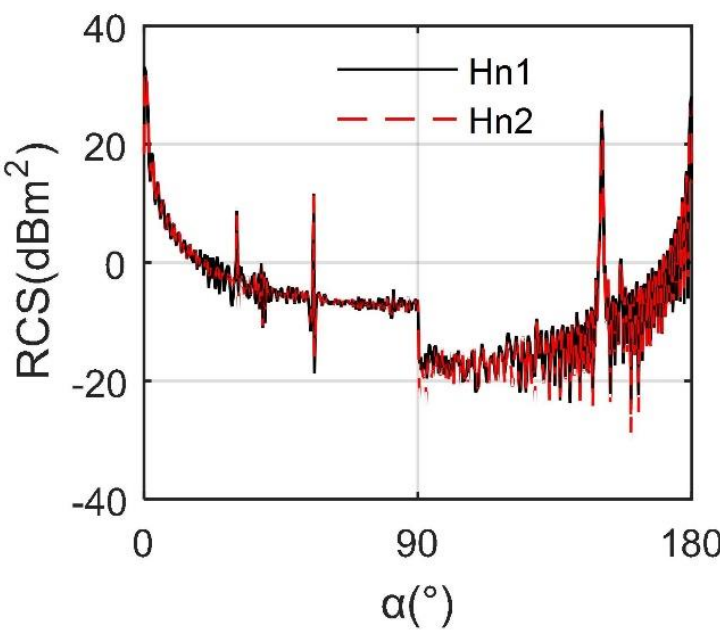

(b) RCS curve

Fig. 13 Effect of exhaust port height on aircraft stealth performance.

\subsection{Effects of $W_{n}$ and $B_{u}$}

Fig. 14 manifests that the difference between RCS and IR curves of aircraft under different $W_{\mathrm{n}}$ is small. For the IR curves, the values of the four curves are relatively large in the range of $0^{\circ} \sim 35^{\circ}$, exceeding $165.7 \mathrm{~W} / \mathrm{sr}$. At this time, the IR of $W_{\mathrm{n} 1}$ and $W_{\mathrm{n} 2}$ is slightly smaller than the other two. Overall, the IR radiation characteristics at these four $W_{\mathrm{n}}$ values are similar, because the opening width of the nozzle is defined on the edge of the tail of the aircraft. For the heat flow that is about to leave the tail of the aircraft, the mixing effect brought by $W_{\mathrm{n}}$ will have little effect on the wall near the outer baffle. For the RCS results, the four curves are very similar, including shape, maximum value, peak size, peak position and number of fluctuations. In the range of $90.75^{\circ} \sim 108.8^{\circ}$, the difference between the four curves is somewhat obvious, because the increase of $W_{\mathrm{n}}$ reduces the surface area and average height of the side baffle outside the spout, which brings changes to the lateral electromagnetic scattering characteristics of the aircraft. As shown in Table 2, the results of the various stealth indicators under different $W_{\mathrm{n}}$ values are very similar, where the maximum difference of the RCS tail indicator is only $0.051 \mathrm{dBm}^{2}$. These also show that the impact of $W_{\mathrm{n}}$ on the overall stealth performance of the aircraft is 
very limited within the given range.



(a) IR curve

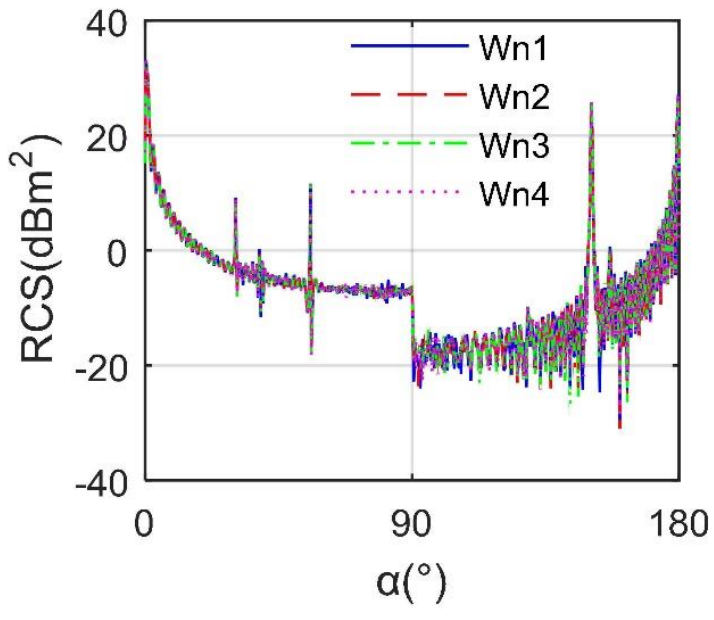

(b) RCS curve

Fig. 14 Comparison of stealth characteristics of aircraft under different $W_{\mathrm{n}}$.

Table 2 Comparison of stealth indicators of aircraft under different $W_{\mathrm{n}}$.

\begin{tabular}{ccccc}
\hline$W_{\mathrm{n}}$ number & 1 & 2 & 3 & 4 \\
\hline$W_{\mathrm{n}}(\mathrm{mm})$ & 800 & 850 & 900 & 950 \\
$\sigma_{\mathrm{m}}\left(\mathrm{dBm}^{2}\right)$ & 10.9572 & 10.9806 & 10.9583 & 10.9544 \\
$\sigma_{\mathrm{t}}\left(\mathrm{dBm}^{2}\right)$ & 12.0435 & 12.0527 & 12.0426 & 12.0017 \\
$I_{\mathrm{n}}(\mathrm{W} / \mathrm{sr})$ & 140.0023 & 139.8559 & 141.4906 & 141.2781 \\
\hline
\end{tabular}

Fig. 15 investigates that different $B_{\mathrm{u}}$ parameters will affect the radar and infrared stealth characteristics of the aircraft, and the impact on the latter is more obvious, where $X_{\mathrm{u} 1}=300 \mathrm{~mm}, X_{\mathrm{u} 2}=400$ $\mathrm{mm}$ and $X_{\mathrm{u} 3}=500 \mathrm{~mm}$. For the RCS results, the three curves are very similar in shape, and all produce large peaks at $0.25^{\circ}, 150.5^{\circ}$ and $179.8^{\circ}$ with the maximum peak reaching $33.04 \mathrm{dBm}^{2}$. For infrared radiation characteristics, the IR curve of $B_{\mathrm{u} 1}$ is obviously larger than that of the other two. The IR values of $B_{\mathrm{u} 1}$ and $B_{\mathrm{u} 2}$ are almost equal in the negative observation angle range, and the IR result of $B_{\mathrm{u} 3}$ in the positive observation angle range is larger than that of $B_{\mathrm{u} 2}$, because the size of the upper baffle outside the nozzle increases and extends outward with the increase of $X_{\mathrm{u}}$, which is helpful to induce the airflow at the back of the fuselage to flow behind the center of the tail flame. This is conducive to the mixing of hot and cold airflow to a certain extent, but it also affects the radiation angle of the tail nozzle. These 
results indicate that $B_{\mathrm{u}}$ is a non-negligible factor affecting the performance of aircraft radar and infrared stealth performance.

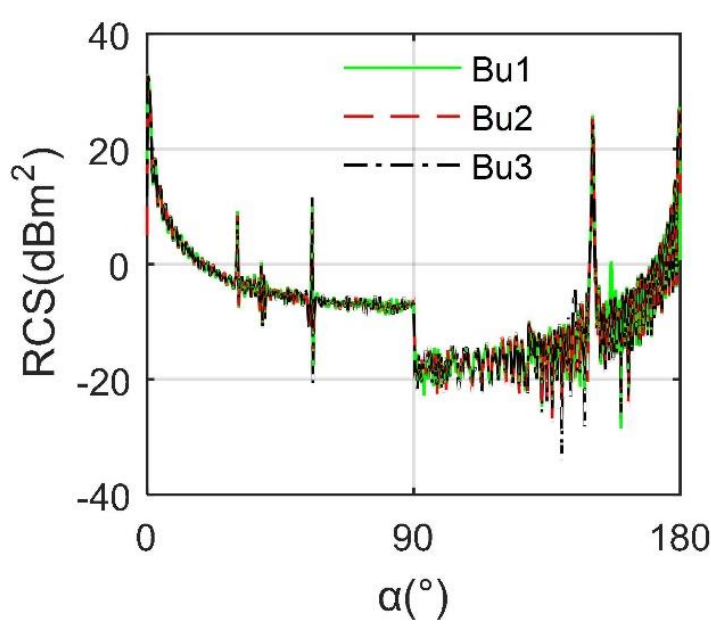

(a) RCS curve

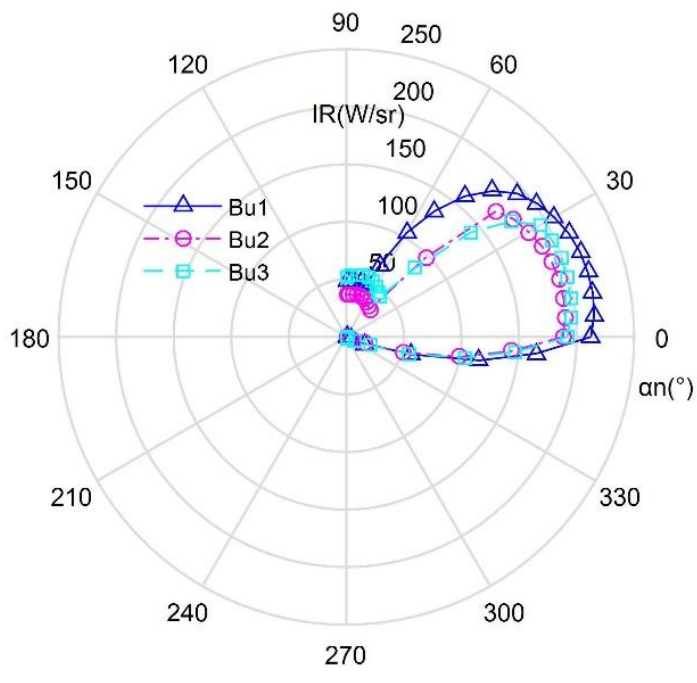

(b) IR curve

Fig. 15 Radar/infrared stealth characteristics of aircraft under different $B_{\mathrm{u}}$.

\subsection{Comprehensive results discussion}

Fig. 16 supports that the three stealth indicators have been improved overall, and the aerodynamic indicator has made a little sacrifice. For the mean RCS of $M^{*}, \sigma_{\mathrm{m}}$ generally maintains a steady downward trend because both ranking factors and priorities are beneficial to this radar stealth indicator. The tail RCS indicator has been greatly reduced, which is conducive to the rearward stealth of the exhaust system. As shown in Table 3, $\sigma_{\mathrm{t}}$ is reduced from greater than $18 \mathrm{dBm}^{2}$ to $12.1045 \mathrm{dBm}^{2}$, the reduction is 6.0763 $\mathrm{dBm}^{2}$. This is mainly because of the reasonable choice of exhaust port model and the optimization of nozzle height. The infrared signature indicator of $M^{*}$ shows a trend of increasing first and then decreasing sharply. Many factors contributed to this process, including the exhaust port model, nozzle series and lower baffle, where $I_{\mathrm{n}}$ indicator has been reduced by $430.2313 \mathrm{~W} / \mathrm{sr}$. The drag performance of the individual fluctuated greatly throughout the process with a maximum difference of $1076.172 \mathrm{~N}$, while the $D_{x}$ indicator of $M^{*}$ increased by $272.9023 \mathrm{~N}$ from $1332.9243 \mathrm{~N}$. Overall, a little drag sacrifice here is 
also acceptable. These results indicate that CDM is satisfactory for improving the radar/infrared stealth characteristics of the aircraft's exhaust system.

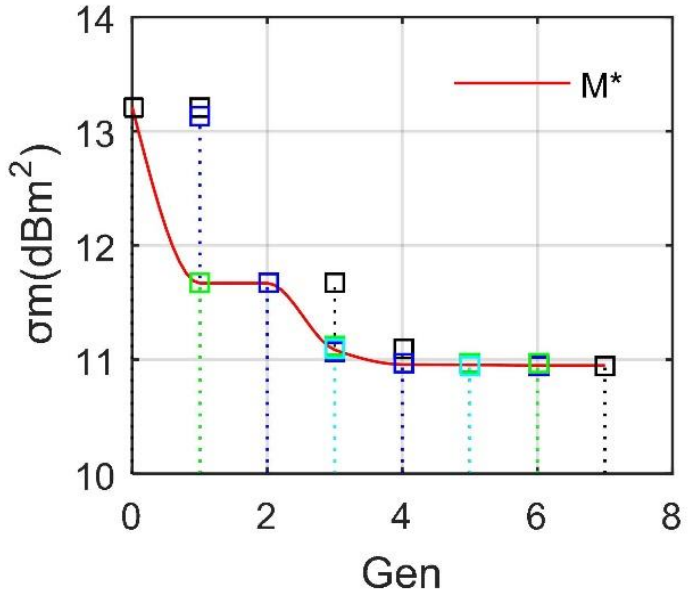

(a) mean RCS indicator

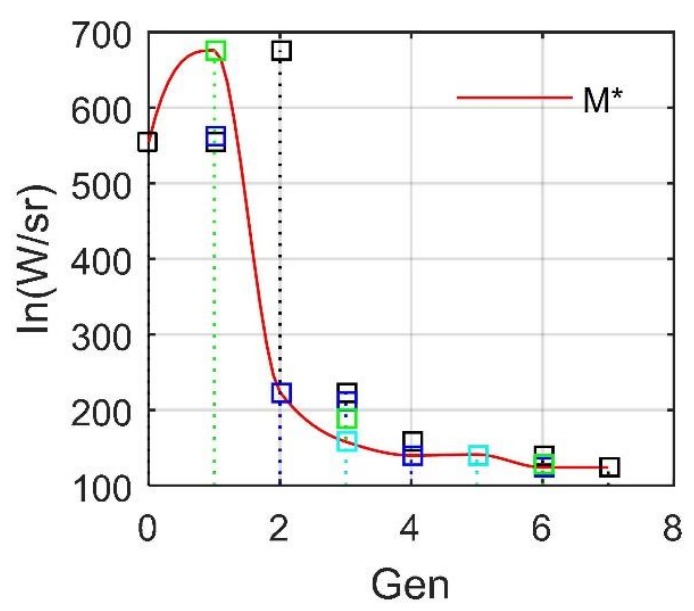

(c) IR indicator

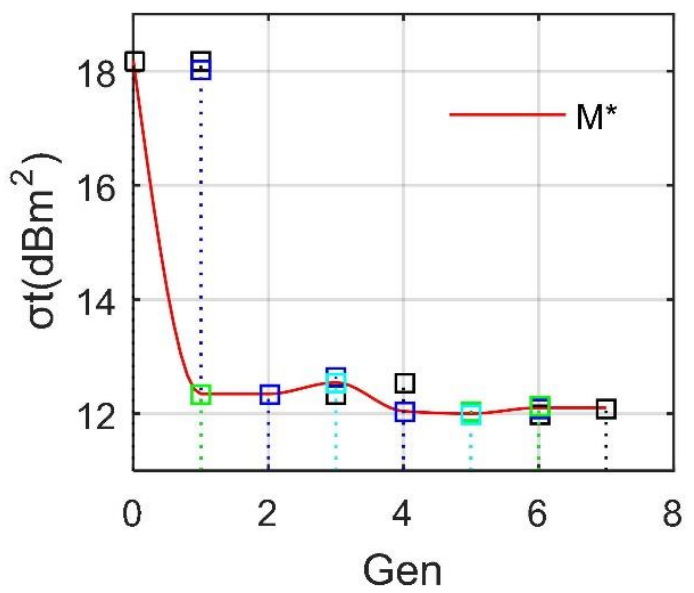

(b) tail RCS indicator

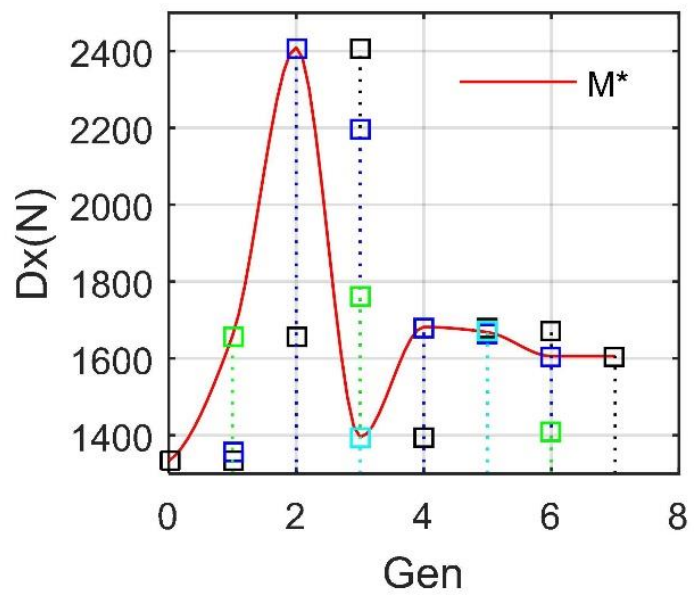

(d) drag indicator

Fig. 16 History chart of various performance indicators.

Table 3 Aircraft main performance comparison before and after CDM.

\begin{tabular}{lllll}
\hline & $\sigma_{\mathrm{m}}\left(\mathrm{dBm}^{2}\right)$ & $\sigma_{\mathrm{t}}\left(\mathrm{dBm}^{2}\right)$ & $I_{\mathrm{n}}(\mathrm{W} / \mathrm{sr})$ & $D_{x}(\mathrm{~N})$ \\
\hline$m_{0}$ & 13.2120 & 18.1808 & 554.3546 & 1332.9243 \\
$M^{*}$ & 10.9492 & 12.1045 & 124.1233 & 1605.8266 \\
\hline
\end{tabular}

The changes in the design parameters or sub-models of the exhaust system are shown in Table 4,

where all the design factors here have been improved under the comprehensive evaluation of CDM. The exhaust port model changed from a simple round tube to a rectangular design with upper and lower 
triangular baffles. The nozzle of the exhaust system was upgraded from single-stage injection to twostage injection. The size of the upper and lower baffles, the height and width of the nozzle have also changed. These results indicate that CDM is clearly effective for the overall design of this aircraft exhaust system.

Table 4 Comparison of main design parameters or sub-models of aircraft before and after CDM.

\begin{tabular}{ccccccc}
\hline & $M_{\mathrm{ex}}$ & $N_{\mathrm{s}}$ & $B_{\mathrm{d}}(\mathrm{mm})$ & $H_{\mathrm{n}}(\mathrm{mm})$ & $W_{\mathrm{n}}(\mathrm{mm})$ & $B_{\mathrm{u}}(\mathrm{mm})$ \\
\hline$m_{0}$ & $M_{\mathrm{ex} 1}$ & 1 & $X_{\mathrm{v}}=500$ & 330 & 800 & $X_{\mathrm{u}}=300$ \\
$M^{*}$ & $M_{\mathrm{ex} 3}$ & 2 & $X_{\mathrm{v}}=1150$ & 300 & 950 & $X_{\mathrm{u}}=400$ \\
\hline
\end{tabular}

Fig. 17 presents that the optimized aircraft model has made significant improvements in surface electromagnetic scattering characteristics and plume temperature field. For the figure 17(a), the cut surface at the tip of the wing, the wall near the tail nozzle and the upper middle part of the fuselage show the most obvious red under the current incident wave, because at this time, the angle between the radar wave and the normal direction of the wing tip is very small, so that the surface here does not have a good ability to deflect the radar wave. The tail nozzle adopts a conventional round tube design, and the nearby end face and baffle form a strong scattering source, that is, a right-angle dihedral angle, which leads to a high RCS level here. For the figure 17(b), at this time, the incident azimuth angle of the radar wave has increased by $2^{\circ}$, and the distribution of strong scattering sources on the surface of the aircraft is still similar compared with the initial model, while the performance of RCS near the tail nozzle has been obviously changed, because the lower baffle of the nozzle of the optimized model adopts a shielding design that blends with the curve of the fuselage tail, the baffle on the side of the nozzle does not form a right-angle dihedral angle with the nozzle end surface, the upper baffle is extended from the surface of the fuselage and cut off with a triangular outer contour, the combined effect of these measures makes the optimized aircraft model have a good low electromagnetic scattering level. For the figure 17(c), it can be found that there is a large-area high-temperature core zone with a temperature range of $696 \mathrm{~K} \sim 841 \mathrm{~K}$ in 
the tail flame outside the nozzle of the initial model, where the static temperature of the space in the exhaust nozzle pipe is basically above $812 \mathrm{~K}$, because the exhaust system at this time uses a single-stage ejection method, which makes the high-temperature wake jet from the center cone unable to be directly cooled until it reaches the edge of the nozzle. For the figure 17(d), it can be noticed that the core area of the plume whose static temperature exceeds $801 \mathrm{~K}$ is confined in the space of the jet pipe, where the static temperature of the tail flame outside the baffle under the nozzle is basically below $648 \mathrm{~K}$, because the optimized exhaust system has a design based on the principle of secondary ejection, coupled with the combined effect of nozzle height, outer width, and upper and lower baffles, the temperature field of the entire aircraft's tail flame is very popular. These results show that the CDM based on the Pareto solution of the ranking factor is feasible and effective to improve the electromagnetic scattering and infrared radiation of this aircraft.

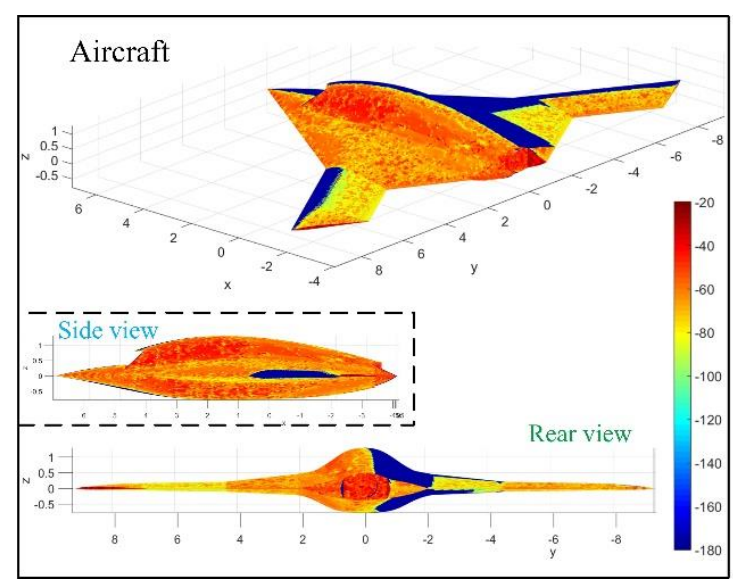

(a) Surface scattering before $\mathrm{CDM}, \beta=0^{\circ}$, $\alpha=127.75^{\circ}$, unit: $\mathrm{dBm}^{2}$

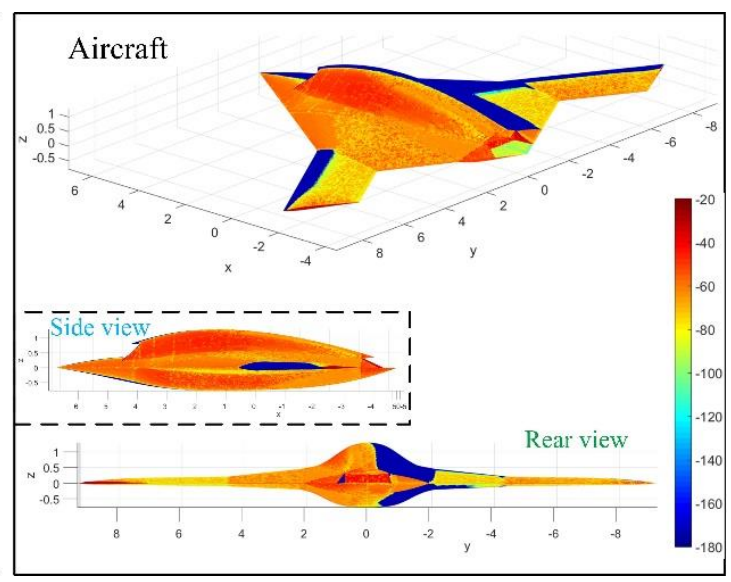

(b) Surface scattering after CDM, $\beta=0^{\circ}$, $\alpha=129.75^{\circ}$, unit: $\mathrm{dBm}^{2}$ 


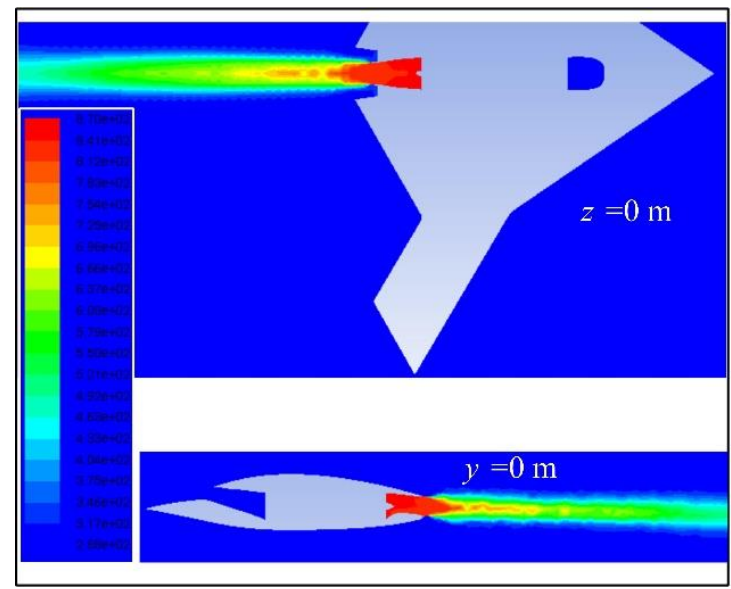

(c) Static temperature before $\mathrm{CDM}$, unit: $\mathrm{K}$

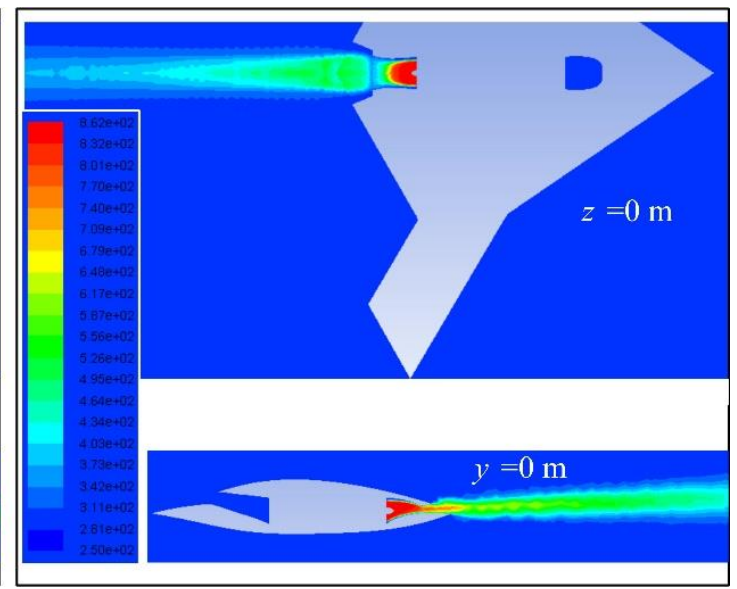

(d) Static temperature after CDM, unit: K

Fig. 17 Performance comparison of the aircraft before and after CDM.

\section{Conclusions}

By studying the radar cross-section and infrared radiation characteristics of this aircraft exhaust system, the relevant parameters and sub-models are fully designed, and the following conclusions can be obtained:

(1) The exhaust port model, lower baffle and nozzle height are the main factors affecting the two RCS indicators of this aircraft model. The design of the upper baffle has a greater impact on the drag indicator than on the other three indicators.

(2) The order that has the greatest influence on the infrared radiation indicator is the nozzle stages, the exhaust port model, the lower baffle, the nozzle outer width and the nozzle height. For the drag indicator, the order is the lower baffle, nozzle stages, exhaust port model, nozzle height and nozzle outer width.

(3) CDM can effectively improve the radar/infrared stealth performance of the aircraft exhaust system while sacrificing some aerodynamic drag. This mainly benefits from the Pareto solution based on ranking factors and priority comparison.

Author contributions: Z.Z. and J.H. conceived the idea; Z.Z. designed the study and carried out the 
computational simulations; Z.Z. and J.H. analyzed the data; Z.Z. wrote the manuscript. All authors reviewed the complete manuscript.

Funding: This work was supported by the project funded by the China Postdoctoral Science Foundation (Grant No. BX20200035, 2020M680005).

Conflicts of Interest: The authors declare no conflicts of interest.

\section{References}

1. Lozano F, Paniagua G. Airfoil leading edge blowing to control bow shock waves. Scientific Reports, 2020, 10(1): 1-18.

2. Chen WJ, Fan W, Zhang Q, et al. Experimental investigation of nozzle effects on thrust and inlet pressure of an air-breathing pulse detonation engine. Chinese Journal of Aeronautics 2012; 25(3): 381-387.

3. Zhou ZY, Huang J, Wu NN. Acoustic and radar integrated stealth design for ducted tail rotor based on comprehensive optimization method. Aerospace Science and Technology 2019; 92: 244-257.

4. Yan XJ, Yang T, Yao HH. Conceptual scheme and key technologies of sixth generation fighters abroad, Aeronautical Science \& Technology 2018; 29(4): 18-26.

5. Li C, Dong H, Zhao K. A balance between aerodynamic and olfactory performance during flight in Drosophila. Nature communications, 2018, 9(1): 1-8.

6. Li M, Bai J, Li L, et al. A gradient-based aero-stealth optimization design method for flying wing aircraft. Aerospace Science and Technology 2019; 92: 156-169.

7. Tan HJ, Guo RW. Design and wind tunnel study of a top-mounted diverterless inlet. Chinese Journal of Aeronautics 2004; 17(2): 72-8.

8. Jiang H, Ang HS. The Analysis of Aerodynamic and Stealth Characteristic of F-35 Fighter. Aircraft 
Design 2010; 30 (6): 1-10.

9. Mirkovic D, Stepanian P M, Kelly J F, et al. Electromagnetic model reliably predicts radar scattering characteristics of airborne organisms. Scientific reports, 2016, 6: 35637.

10. Ma Y, Pan ZX, Luo L. X-47B flying wing aerodynamic configuration analysis. Aeronautical Science \& Technology 2014; 25 (12): 1-4.

11. Murfitt S L, Allan B M, Bellgrove A, et al. Applications of unmanned aerial vehicles in intertidal reef monitoring. Scientific reports, 2017, 7(1): 1-11.

12. Yue K, Liu W, Li G, et al. Numerical simulation of RCS for carrier electronic warfare airplanes. Chinese Journal of Aeronautics 2015; 28(2): 545-555.

13. Huang Z, Jiang W, Zhang Y. Brief introduction to infrared stealth and detection technology of aircraft. Infrared 2017; 38(8): 1-7, 43.

14. Qu ZJ, Zhang EL, Zhou FF. Modeling infrared radiation characteristics for F35 stealth fighter. Infrared Technology 2014; 36(11): 920-925.

15. Ma GJ, Zhang YF, Lai DX. Numerical research on infrared radiation of engine exhaust system. Aricraft Design 2015; 35(1): 30-34.

16. Zhang Y, Chen J, Shen L. Real-time trajectory planning for UCAV air-to-surface attack using inverse dynamics optimization method and receding horizon control. Chinese Journal of Aeronautics 2013; 26(4): 1038-1056.

17. Jia QL. Numerical calculation of IR radiation for unmanned aerial vehicle. Laser \& optoelectronics progress 2014; 51: 071201 .

18. Li X, Yang QZ, Chen LH, et al. Numerical simulation of infrared characteristics of aero-engine exhaust nozzle. Advances in Aeronautical Science and Engineering 2013; 4(1): 126-133. 
19. Cheng W, Wang W, Zhou L, et al. Infrared signature of serpentine nozzle with engine swirl, Aerospace Science and Technology 2019; 86: 794-804.

20. Zhou ZY, Huang J, Yi MX. Comprehensive optimization of aerodynamic noise and radar stealth for helicopter rotor based on Pareto solution. Aerospace Science and Technology 2018; 82: 607-619.

21. Cheng X, Sun M. Wing-kinematics measurement and aerodynamics in a small insect in hovering flight. Scientific reports, 2016, 6: 25706.

22. Sun ZJ, Liu L, Li H, et al. Analysis on aerodynamic / stealth synthetical performance of flying wing UAV. Flight Dynamics 2018; 36 (3): 15-18, 27.

23. Wang B, Cong W, Wang CZ, et al. Infrared radiation characteristics calculation and infrared stealth effect analysis of stealth fighter, Transactions of Beijing Institute of Technology 2019; 39(4): 365371.

24. Han J S, Han J H. A contralateral wing stabilizes a hovering hawkmoth under a lateral gust. Scientific Reports, 2019, 9(1): 1-13.

25. Zhou ZY, Huang J, Wu NN. Acoustic and radar integrated stealth design for ducted tail rotor based on comprehensive optimization method. Aerospace Science and Technology 2019; 92: 244-257.

26. Anagnostopoulos KA, Charalambopoulos A, Fotiadis DI. Scattering of electromagnetic spherical waves by a buried spheroidal perfect conductor. International Journal of Engineering Science, 2003, 41(11): 1239-1266.

27. Zhen ZY, Zhu P, Xue YX, et al. Distributed intelligent self-organized mission planning of multiUAV for dynamic targets cooperative search-attack. Chinese Journal of Aeronautics 2019; 32(12): 2706-2716.

28. Liu J, Yue H, Lin J, et al. A simulation method of aircraft infrared signature measurement with 
subscale models. Procedia Computer Science 2019; 147: 2-16.

29. Iemma U, Palma G. Design of metacontinua in the aeroacoustic spacetime. Scientific Reports, 2020, 10(1): 18192.

30. Zhu H J, Meng X G, Sun M. Forward flight stability in a drone-fly. Scientific Reports, 2020, 10(1): 1975. 




\section{Figure 1}

Schematic diagram of radar/infrared integrated stealth design of aircraft exhaust system.



\section{Figure 2}

Flow chart of the comprehensive design method. 


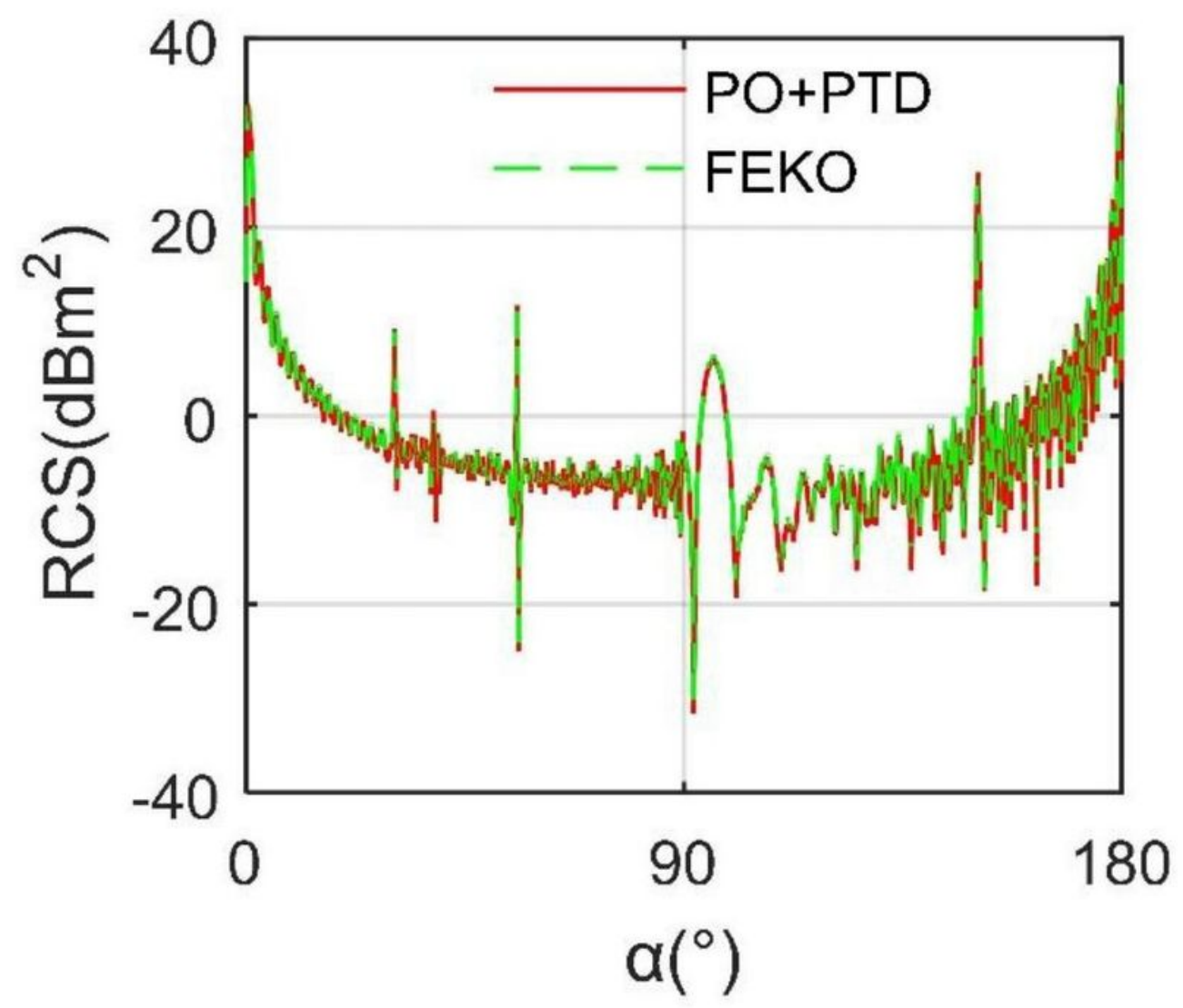

Figure 3

Verification of RCS calculation method on $m 0, \beta=0^{\circ}, f R=10 \mathrm{GHz}$. 


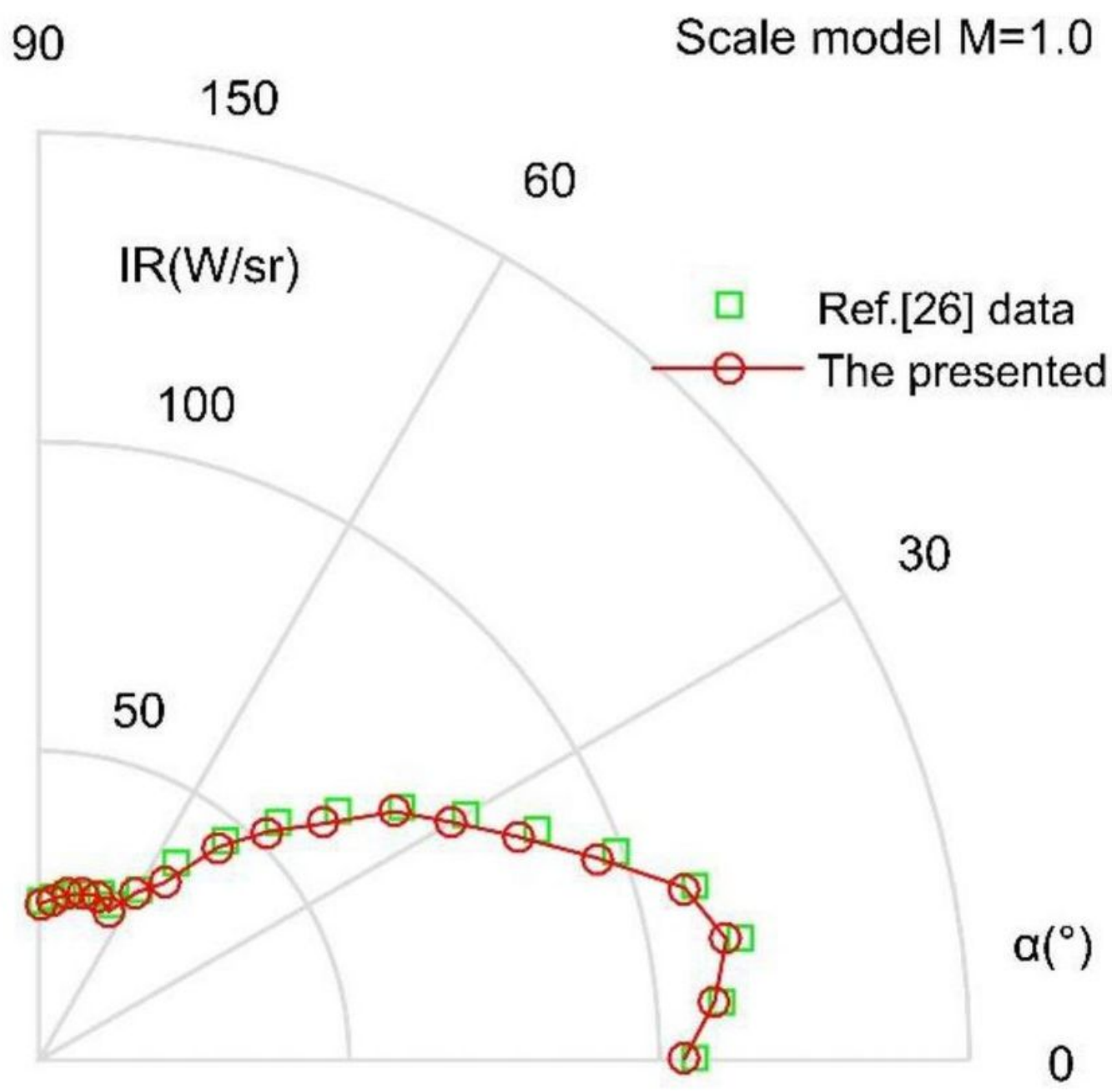

Figure 4

Verification of IR signature calculation method, 3-5 $\mu \mathrm{m}$ wavelength band. 


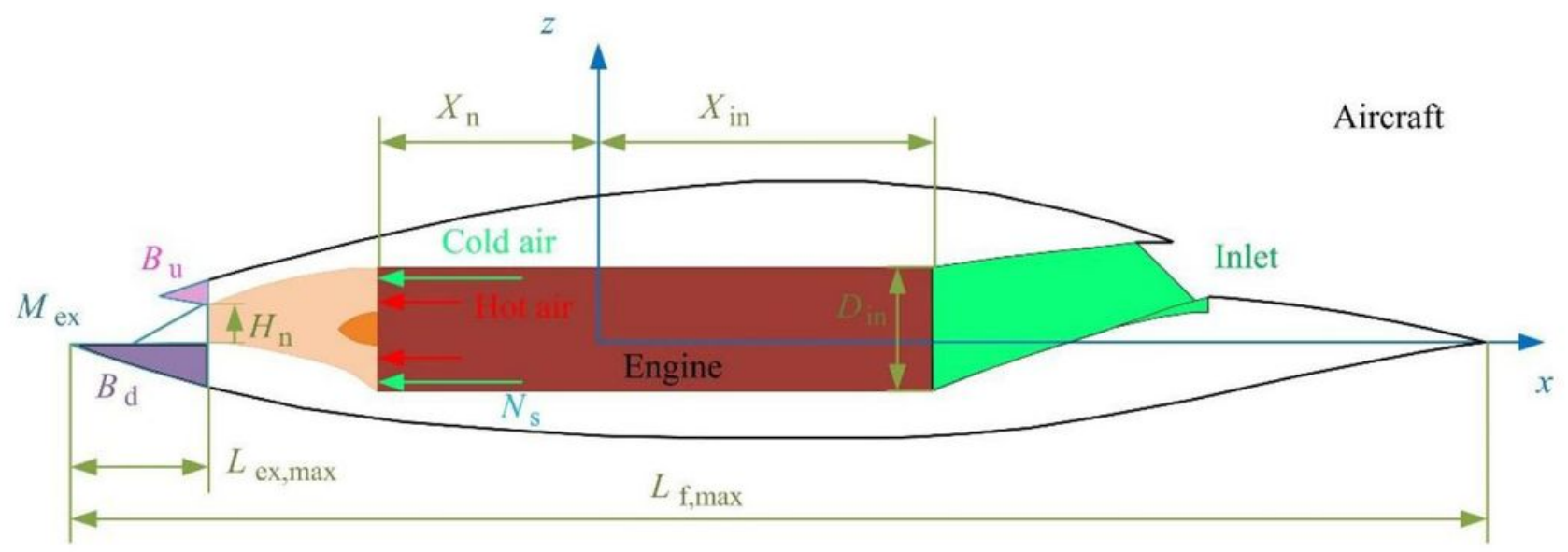

Figure 5

Aircraft design draft and parameter variables.

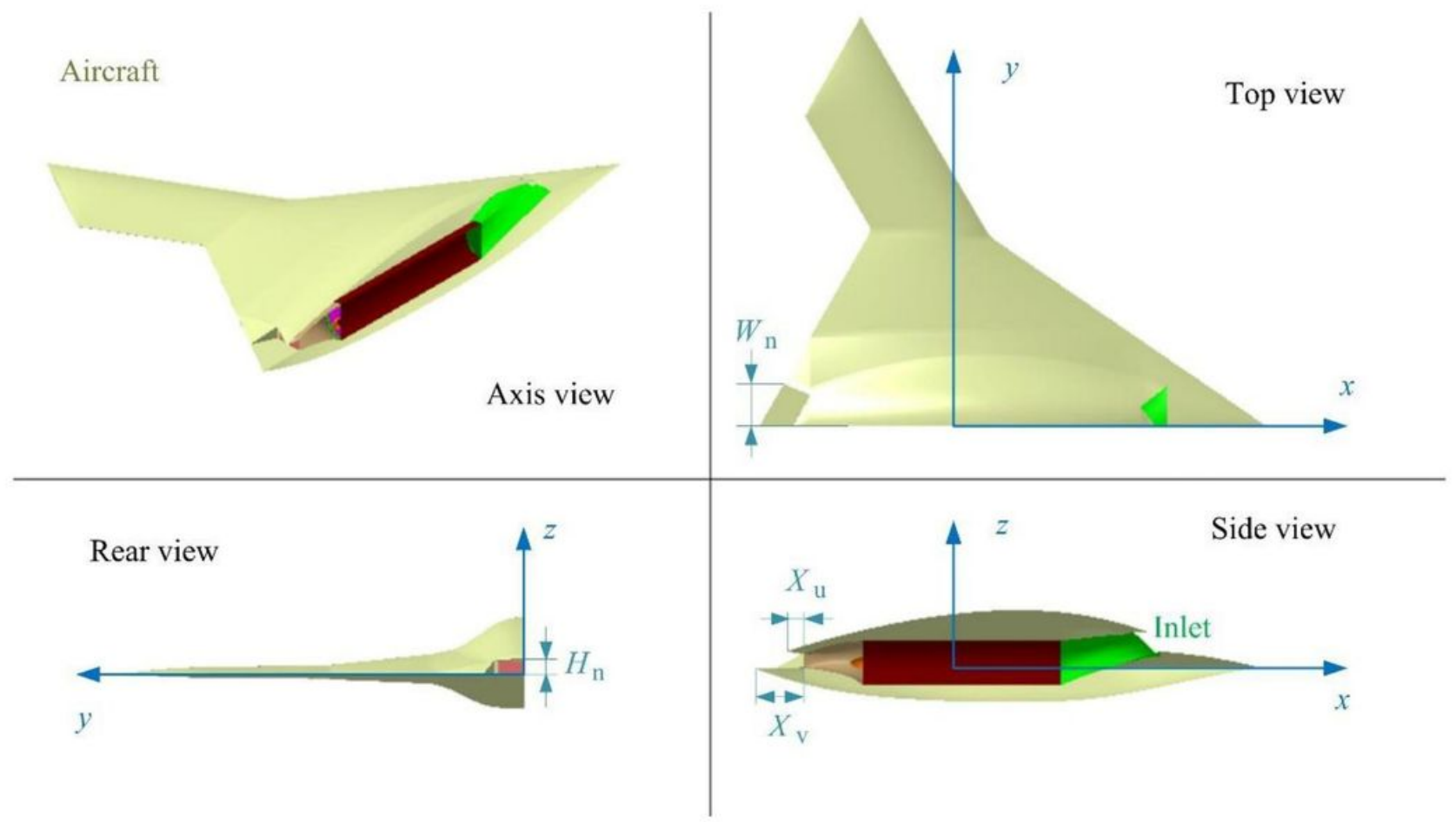

Figure 6

Three-view display of aircraft geometric model. 


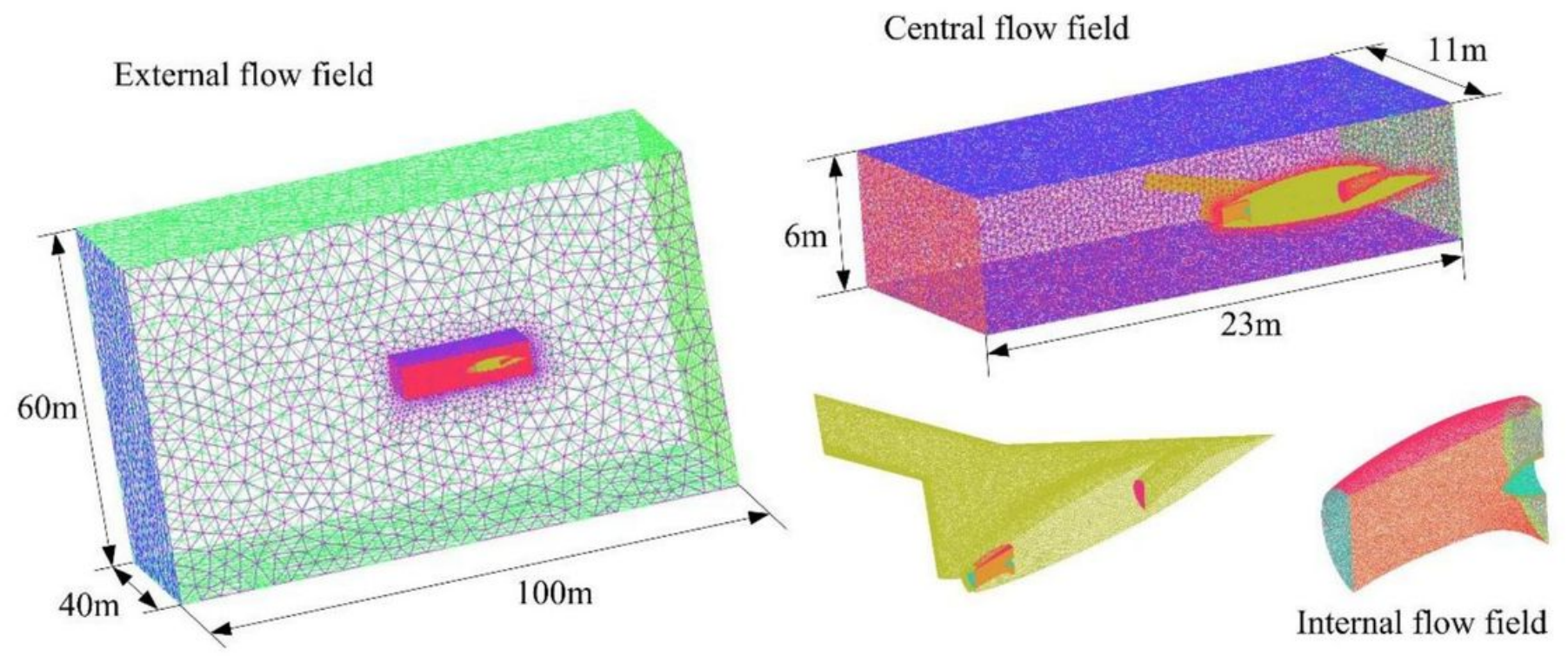

Figure 7

Aircraft exhaust system flow field construction and grid division.
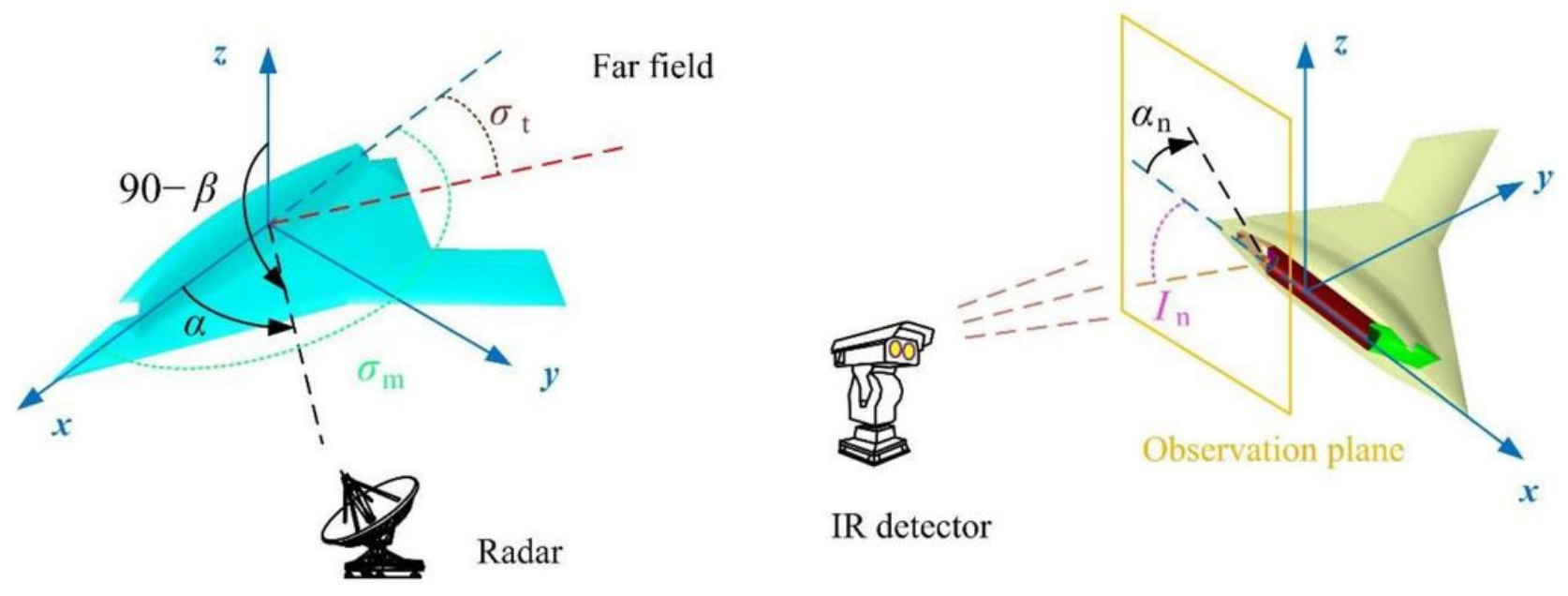

IR detector

Figure 8

Observation field setting of aircraft radar cross section and IR signature, $\beta=0^{\circ}$. 


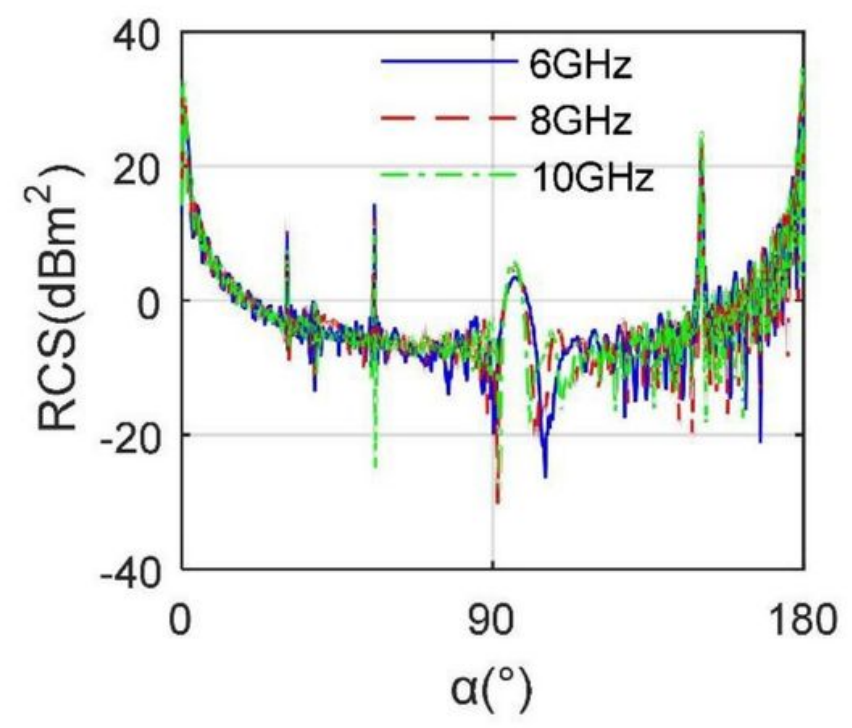

(a) RCS



(b) mean RCS

Figure 9

RCS of aircraft model under different radar wave frequencies, $\beta=0^{\circ}$.

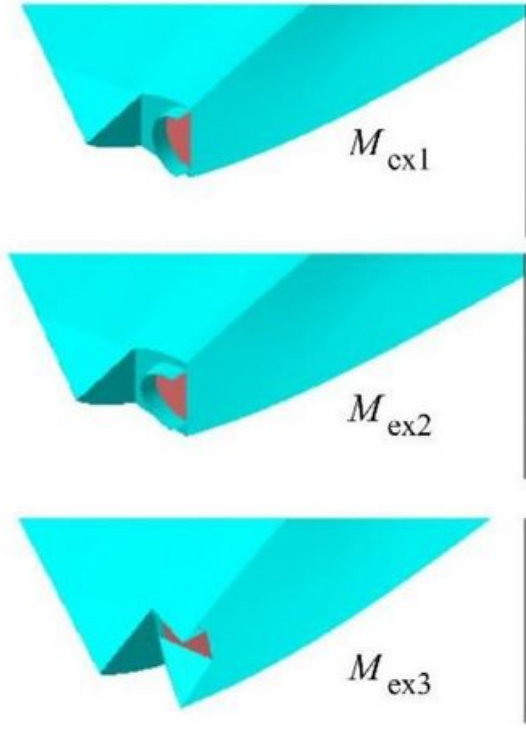

(a) $M_{\mathrm{ex}}$

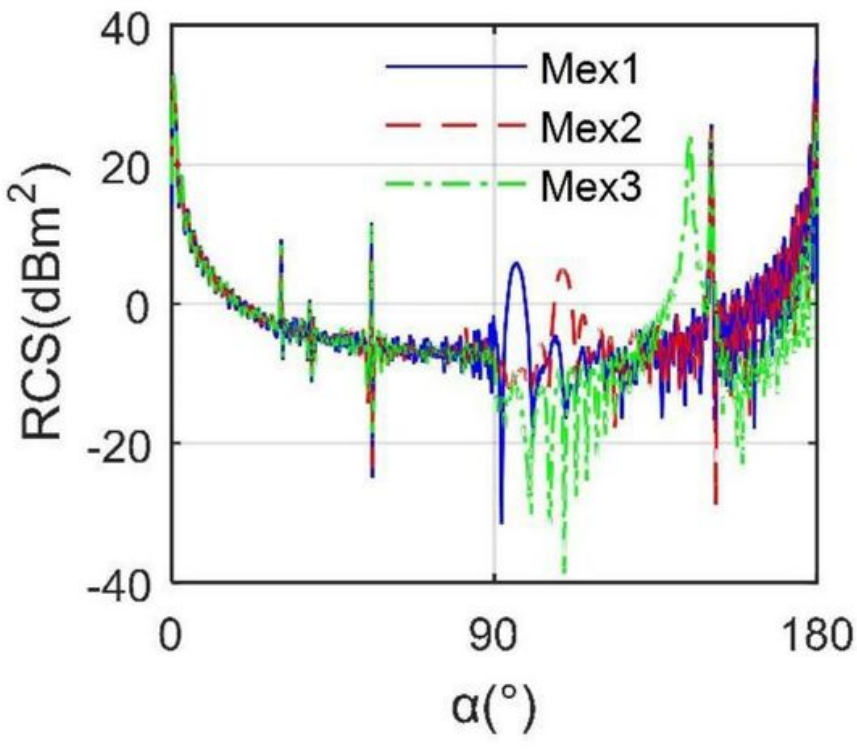

(b) RCS

Figure 10

RCS of aircraft under different exhaust port models, $f R=10 \mathrm{GHz}, \beta=0^{\circ}$. 


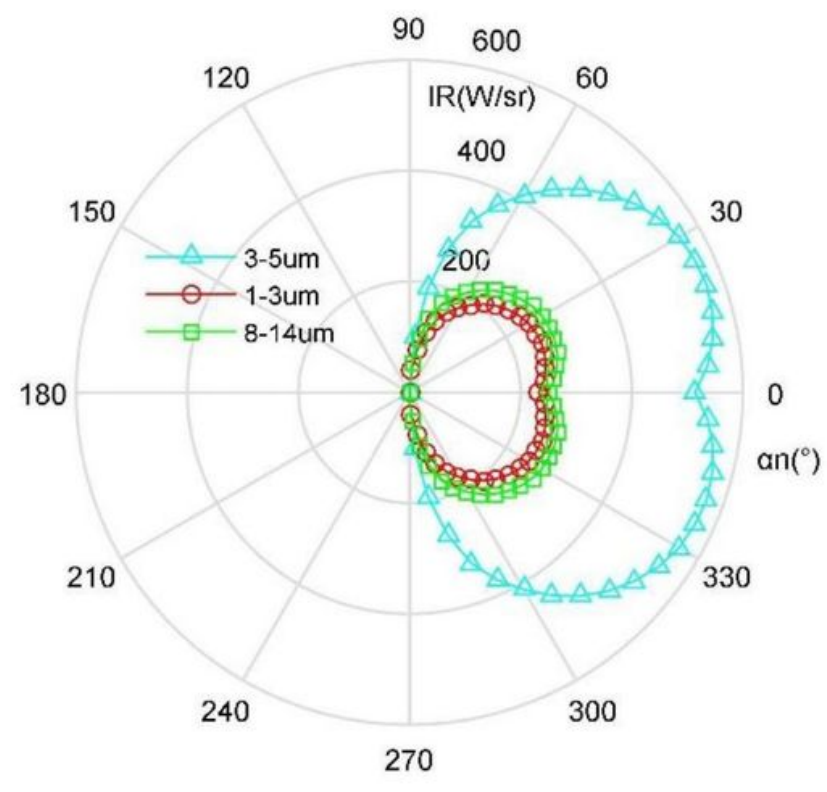

(a) $m_{0}, N_{\mathrm{s}}=1$

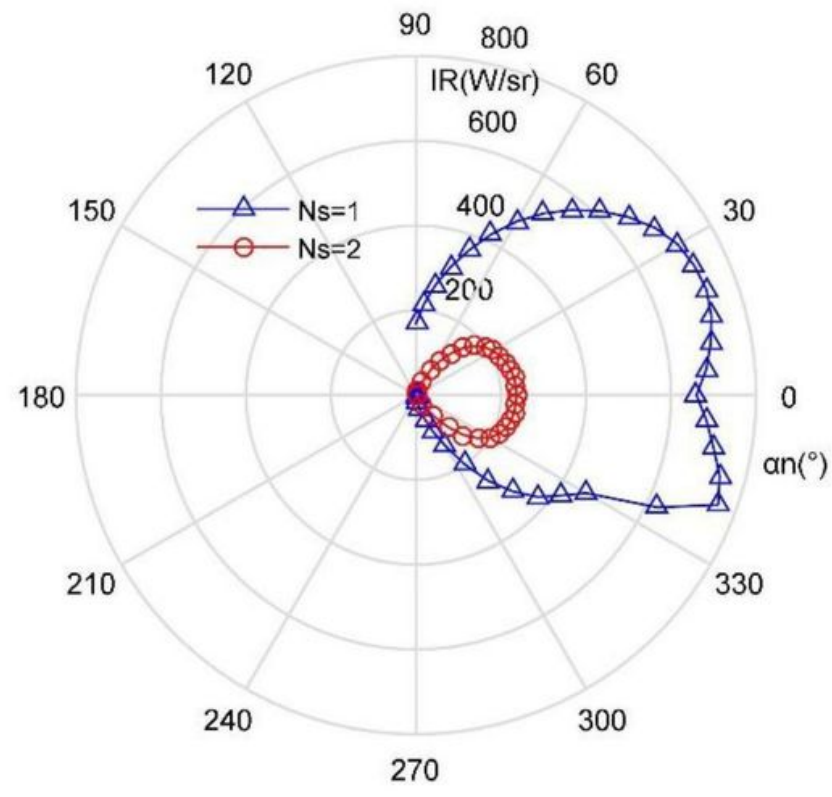

(b) $M_{\mathrm{ex} 3}, 3 \sim 5 \mu \mathrm{m}$

\section{Figure 11}

Aircraft IR signature under different wave bands and Ns.

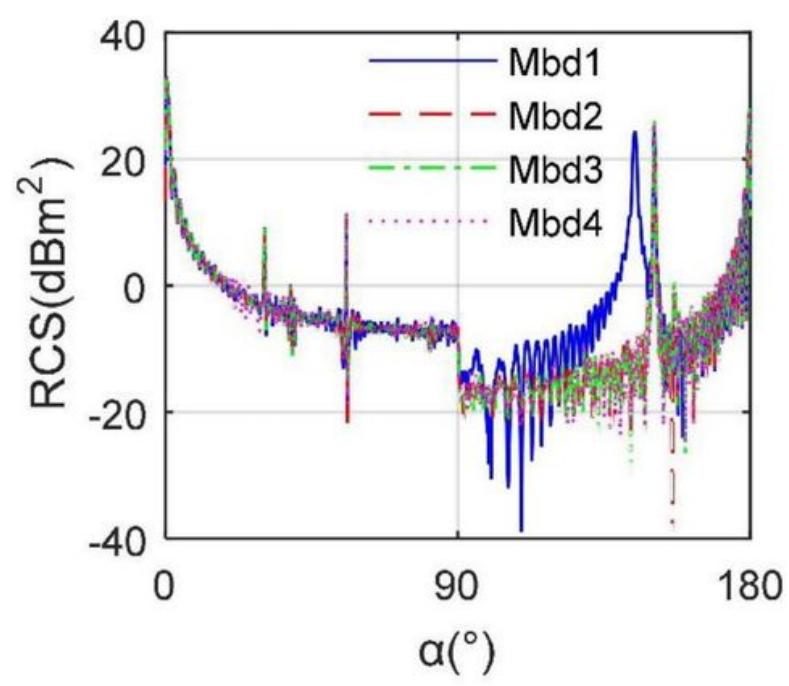

(a) RCS

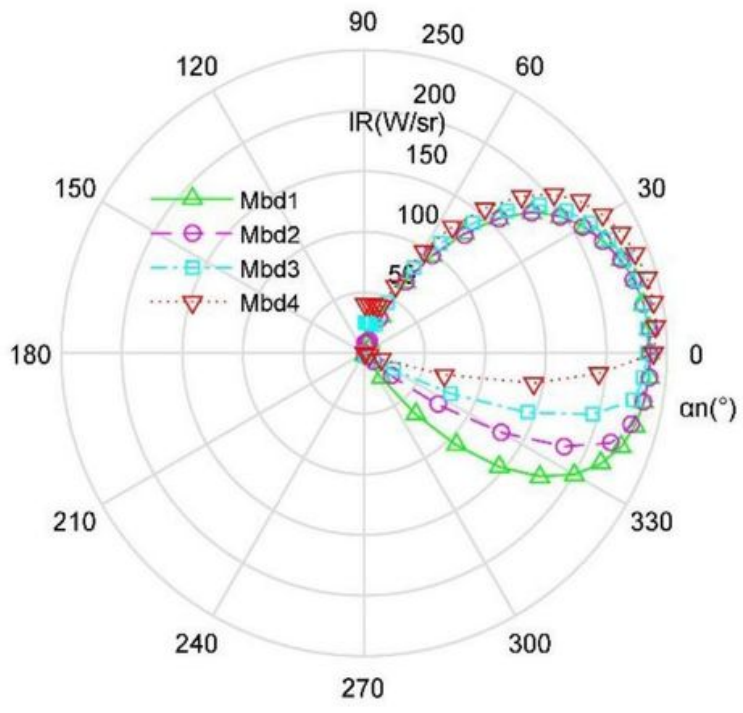

(b) IR curve

Figure 12

Comparison of stealth characteristics of aircraft under different Mbd models. 


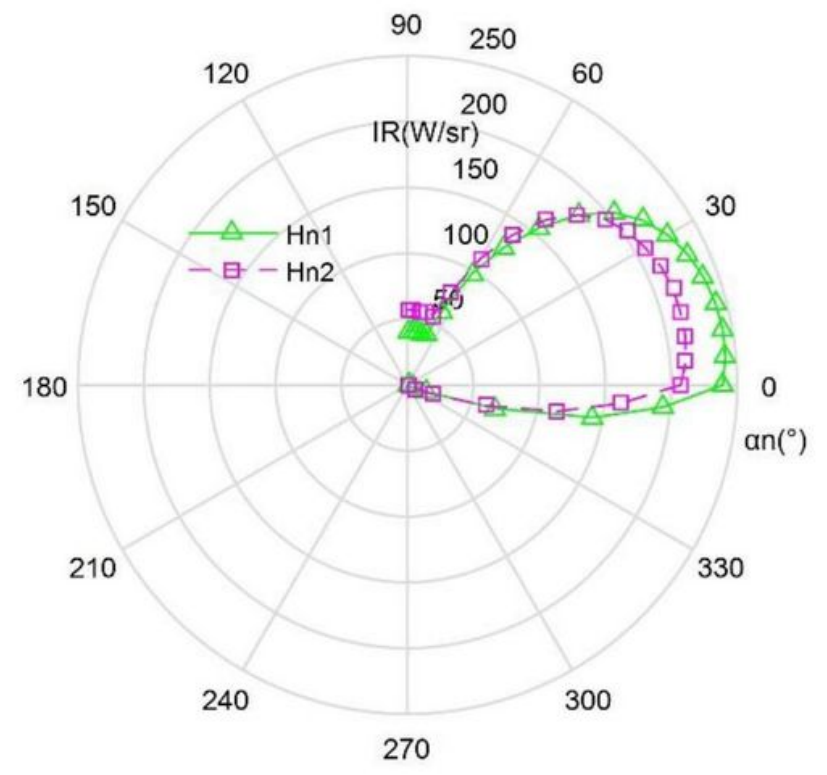

(a) IR curve

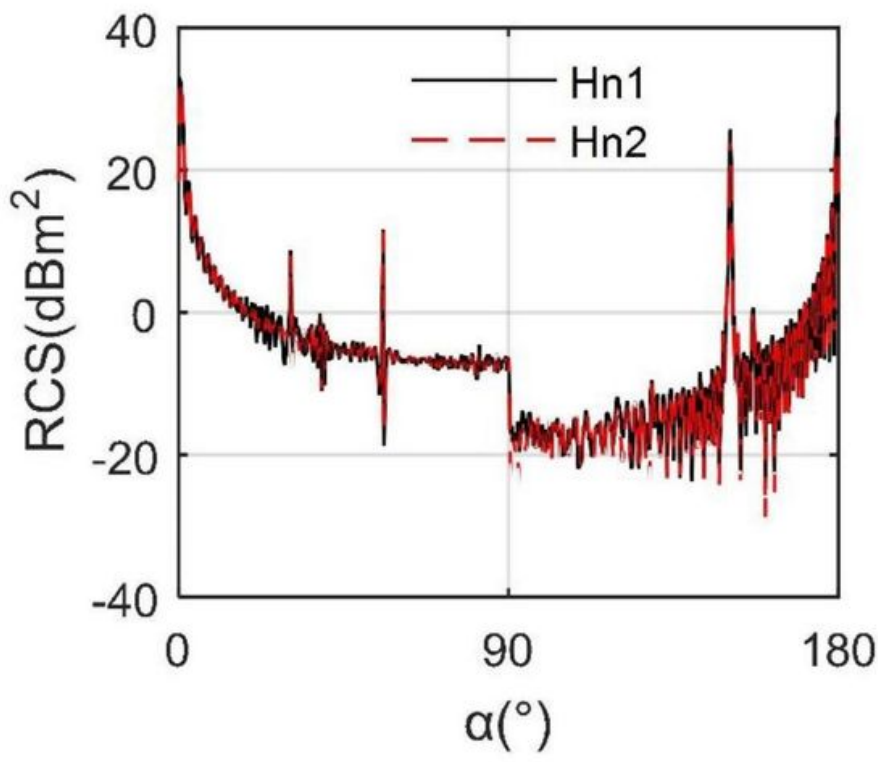

(b) RCS curve

\section{Figure 13}

Effect of exhaust port height on aircraft stealth performance.

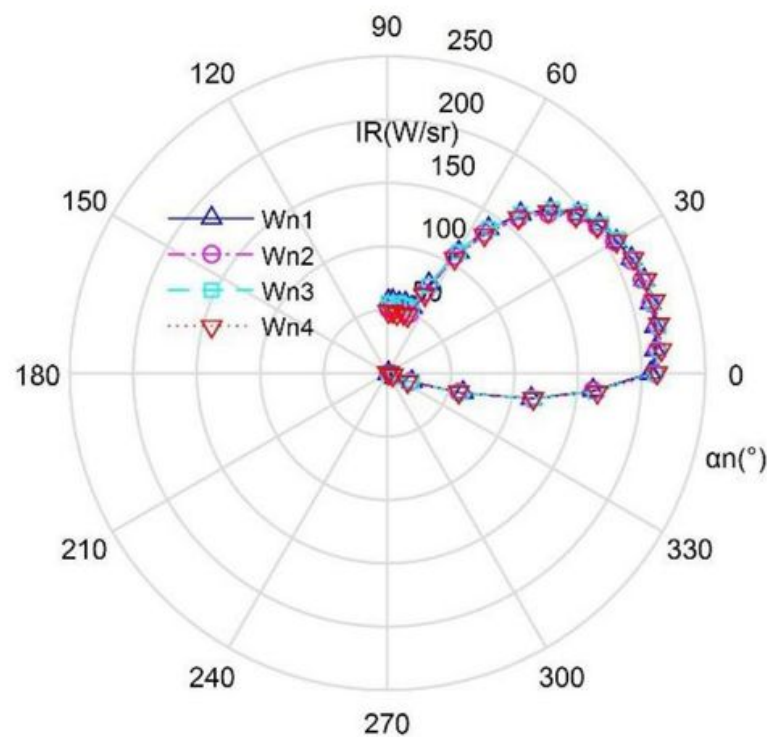

(a) IR curve

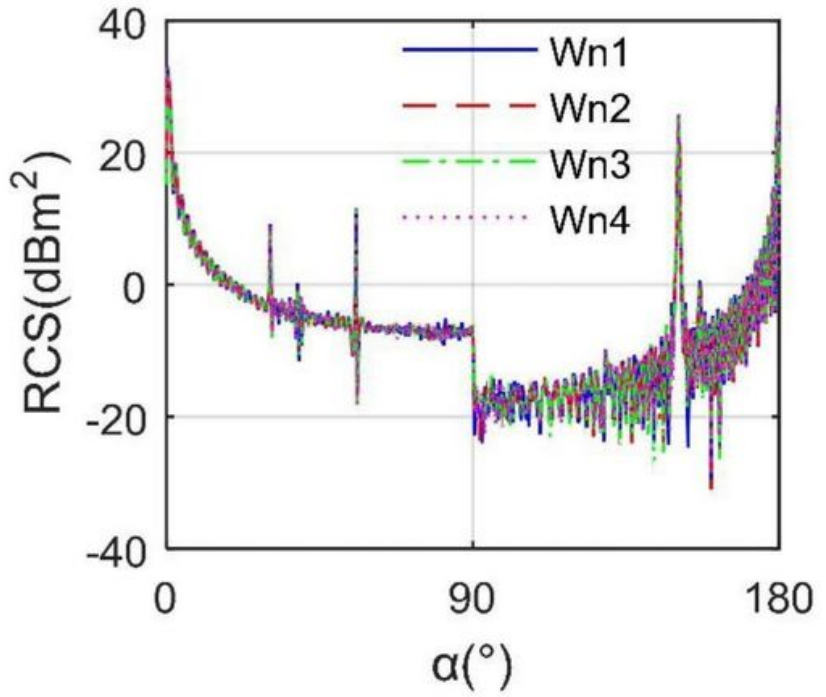

(b) RCS curve

Figure 14

Comparison of stealth characteristics of aircraft under different Wn. 


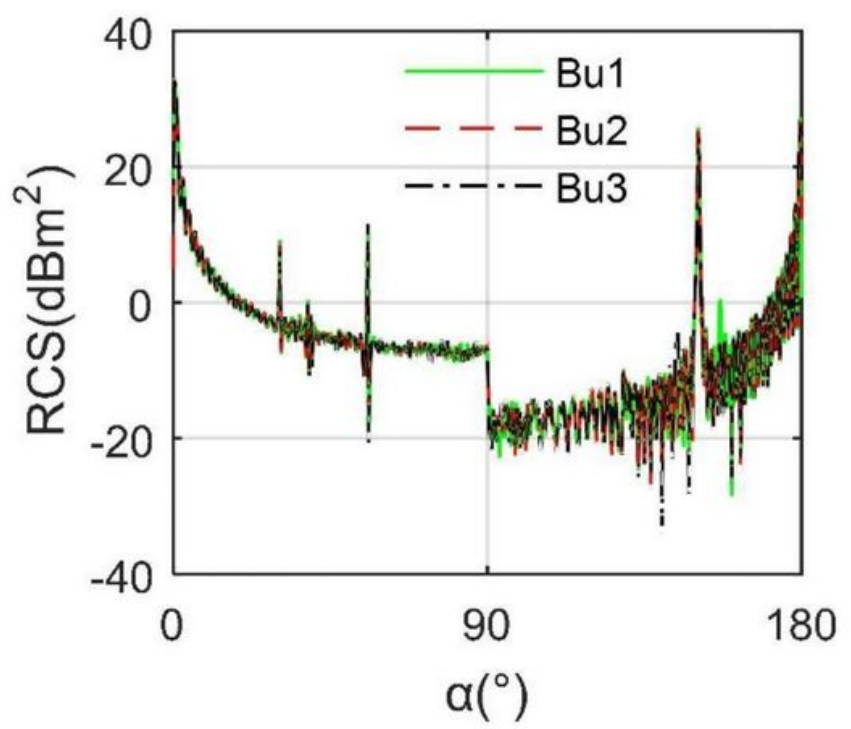

(a) RCS curve

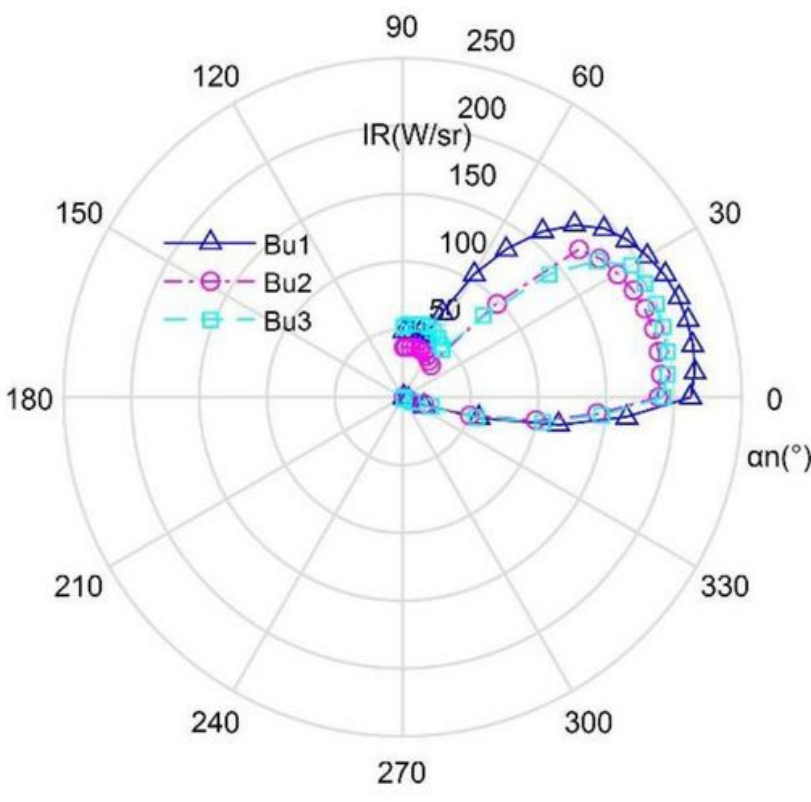

(b) IR curve

Figure 15

Radar/infrared stealth characteristics of aircraft under different Bu. 




(a) mean RCS indicator

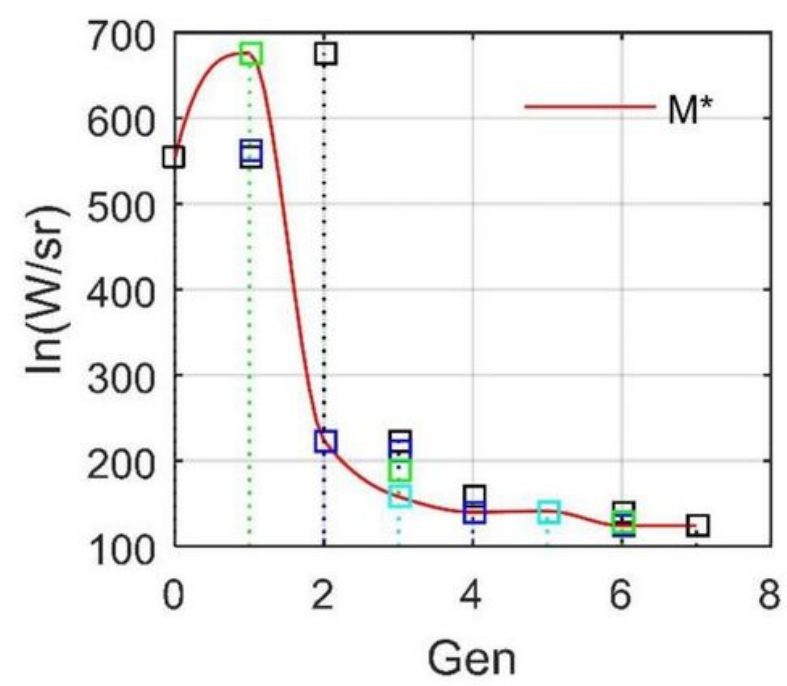

(c) IR indicator

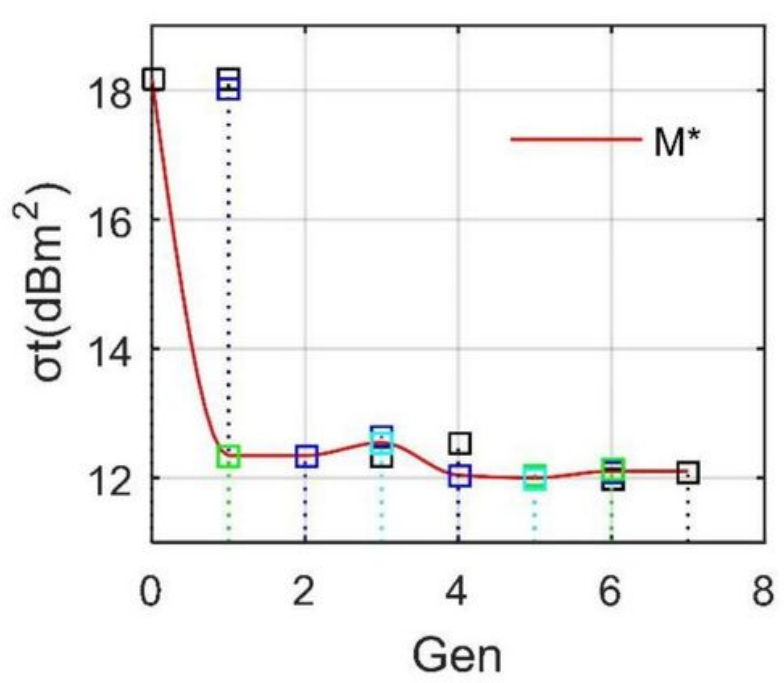

(b) tail RCS indicator

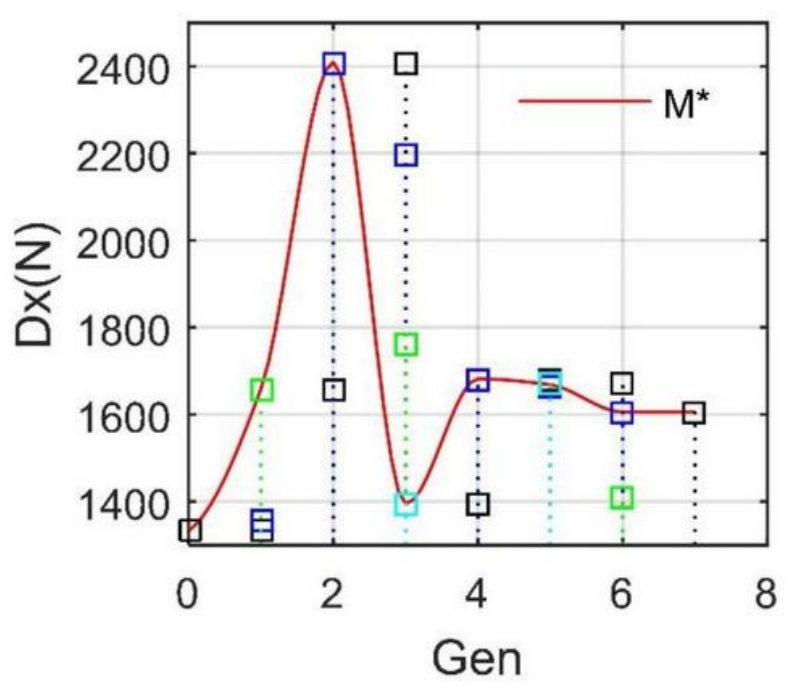

(d) drag indicator

Figure 16

History chart of various performance indicators. 


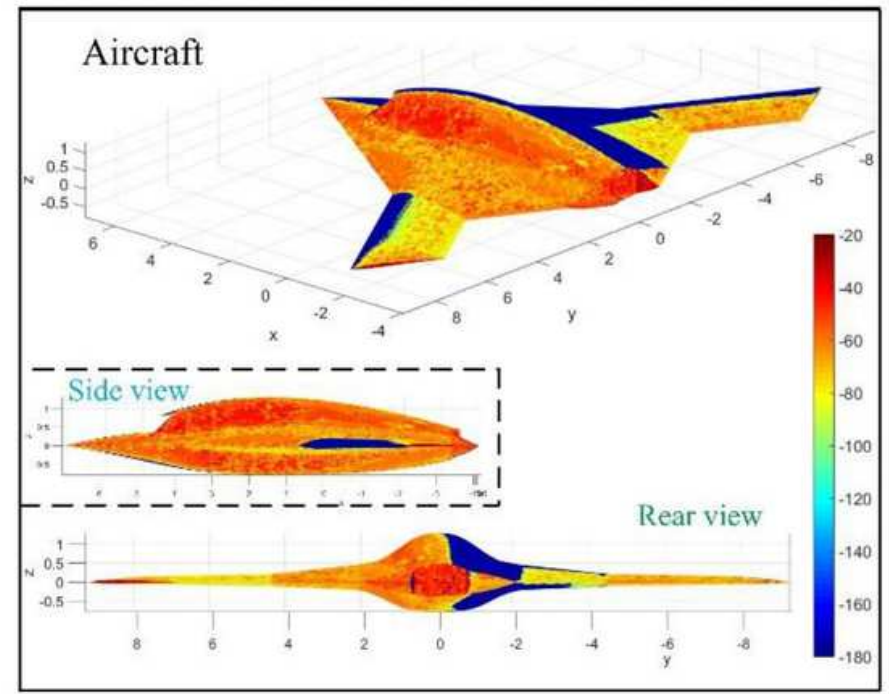

(a) Surface scattering before $\mathrm{CDM}, \beta=0^{\circ}$, $\alpha=127.75^{\circ}$, unit: $\mathrm{dBm}^{2}$



(c) Static temperature before CDM, unit: $\mathrm{K}$

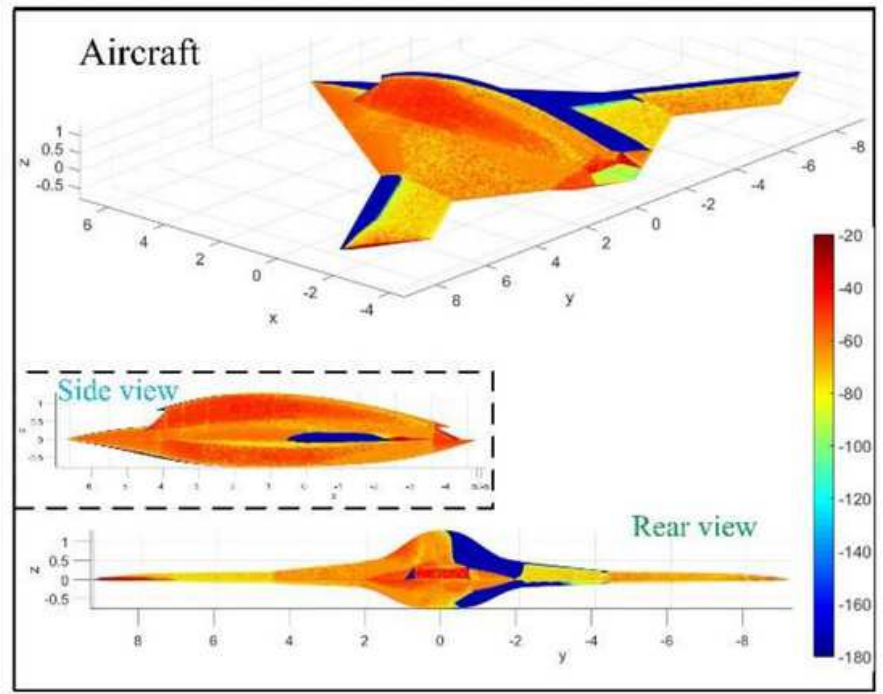

(b) Surface scattering after $\mathrm{CDM}, \beta=0^{\circ}$, $\alpha=129.75^{\circ}$, unit: $\mathrm{dBm}^{2}$

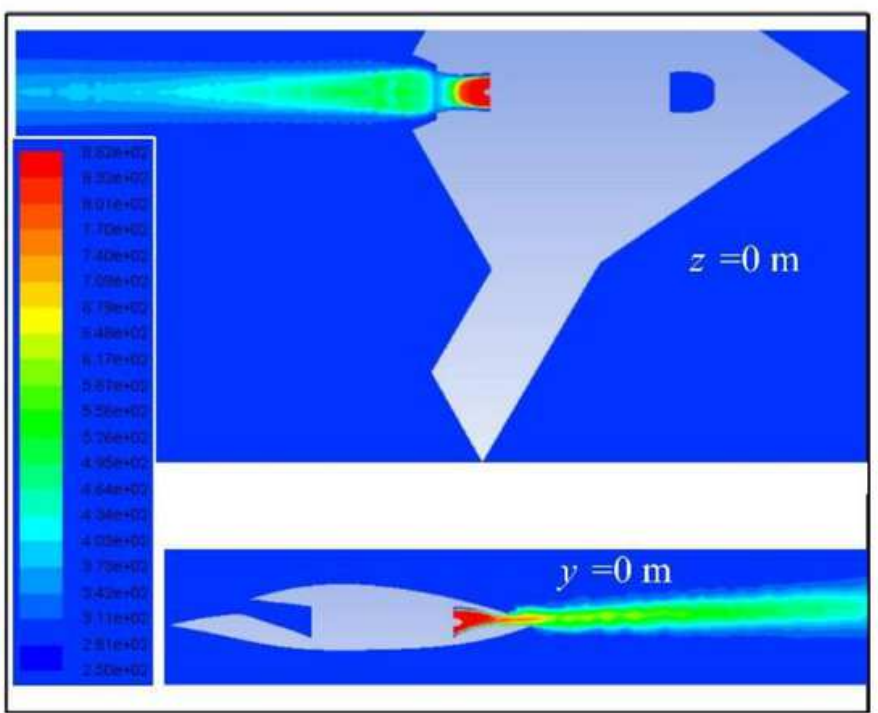

(d) Static temperature after CDM, unit: K

\section{Figure 17}

Performance comparison of the aircraft before and after CDM. 